\begin{tabular}{|c|l|}
\hline Title & An inverse dynamical problem for connected beams \\
\hline Author(s) & Morassi, A ntonino; Nakamura, Gen; Sini, Mourad \\
\hline Citation & Hokkaido University Preprint Series in Mathematics, 648, 1-27 \\
\hline Issue Date & 2004-05-20 \\
\hline DOI & 10.14943/83801 \\
\hline Doc URL & http://hdl.handle.net/2115/69455 \\
\hline Type & bulletin (article) \\
\hline File Information & pre648.pdf \\
\hline
\end{tabular}

Instructions for use 


\title{
An inverse dynamical problem for connected beams
}

\author{
A. Morassi * \\ G. Nakamura ${ }^{\dagger}$ \\ M. Sini ${ }^{\ddagger}$ \\ May 20, 2004
}

\begin{abstract}
This paper deals with a dynamical inverse problem for a composite beam formed by two connected beams. The vibrations of the composite beam are governed by a differential system where a coupling takes place between longitudinal and bending motions. In this paper, we neglect bending motions and we only deal with the longitudinal motions. These motions are governed by a two-by-two second order system coupled in the lower order terms by the shearing stiffness coefficient, which models the connection between the two beams and which contains direct information on the integrity of the system. We prove that the shearing stiffness coefficient can be reconstructed from the frequency response function of the system evaluated at one end of the beam.
\end{abstract}

Key words: inverse problems, composite beams, vibrations.

\section{Introduction}

In this paper we address the following problem of nondestructive testing: To determine, in a steel-concrete composite beam, the possible presence of a damage from dynamic measurements of time-histories of forces and displacements taken at one end-section of the beam.

Steel-concrete composite beams are obtained by connecting two beams, a metallic one and a reinforced concrete (r.c.) beam, by means of small metallic elements which are welded on the

*Department of Georesources and Territory, University of Udine, 33100 Udine, Italy (Email: antonino.morassi@uniud.it) Partially supported by Grant-in-Aid for Scientific Research (B)(2)(No. 14340038) of Japan Society for Promotion of Science and by MIUR grant 2003082352.

†Department of Mathematics, Hokkaido University, Sapporo 060-0810, Japan. (Email: gnaka@math.sci.hokudai.ac.jp) Partially supported by Grant-in-Aid for Scientific Research (B)(2)(No. 14340038) of Japan Society for Promotion of Science.

${ }_{\ddagger}^{\ddagger}$ Department of Mathematics, Hokkaido University, Sapporo 060-0810, Japan. (Email: sini@math.sci.hokudai.ac.jp ) Partially supported by Grant-in-Aid for Scientific Research (B)(2)(No. 14340038) of Japan Society for Promotion of Science. 
top flange of the metallic beam and immersed in the concrete, in order to hinder sliding on the concrete-steel interface.

This structural solution increases the stiffness in the whole beam and, therefore, is commonly employed in designing large structures, such as bridge decks or long span floor beams, when a severe control of deformability under important loads is required. For this class of structures, the connection is the structural component having to bear the major consequences of stress and fatigue during service. It follows that structural damages usually involve a deterioration of the connection, causing a decrease of the overall rigidity of the system and a reduction of its resistance. The inaccessibility of the connection from the exterior makes direct inspection difficult and, therefore, finding nondestructive assessment techniques that allow checks on the integrity of the connection would be of great importance for practical purposes.

In this paper a nondestructive dynamic technique of damage detection based on dynamic measurements is presented. Although a very large literature on the static behavior of steelconcrete composite structures is nowadays available, see, for example, the book by Johnson $[\mathrm{J}]$ for an exhaustive and well-grounded bibliography, one of the first experimental-analytical investigations on the dynamical behavior of composite beams has been recently presented in $[\mathrm{BMW}$. A one-dimensional mechanical model of the small vibrations of a composite beam was developed in that paper. The elements connecting the steel beam and the r.c. slab were described by means of a strain energy density function defined throughout the longitudinal axis and the two beams were forced to maintain equal transverse displacements, see also $[\mathrm{DM}]$ for a more refined model. In mathematical terms, the small free vibrations of a composite beam of length $L$ are governed by the differential system

$$
\begin{cases}\frac{\partial}{\partial x}\left(a_{1} \frac{\partial u_{1}}{\partial x}\right)+k\left(u_{2}-u_{1}+\frac{\partial v}{\partial x} e_{s}\right)=\rho_{1} \frac{\partial^{2} u_{1}}{\partial t^{2}} \quad(x, t) \in(0, L) \times(0, T), \\ \frac{\partial}{\partial x}\left(a_{2} \frac{\partial u_{2}}{\partial x}\right)-k\left(u_{2}-u_{1}+\frac{\partial v}{\partial x} e_{s}\right)=\rho_{2} \frac{\partial^{2} u_{2}}{\partial t^{2}} \quad(x, t) \in(0, L) \times(0, T), \\ -\frac{\partial^{2}}{\partial x^{2}}\left(j \frac{\partial^{2} v}{\partial x^{2}}\right)+\frac{\partial}{\partial x}\left(k\left(u_{2}-u_{1}+\frac{\partial v}{\partial x} e_{s}\right) e_{s}\right)+\frac{\partial}{\partial x}\left(k e_{c}^{2} \frac{\partial v}{\partial x}\right)=\rho \frac{\partial^{2} v}{\partial t^{2}} \quad(x, t) \in(0, L) \times(0, T) .\end{cases}
$$

In what follows, we shall concern with homogeneous initial conditions, that is

$$
\left.u_{1}\right|_{t=0}=\left.u_{2}\right|_{t=0}=\left.v\right|_{t=0}=0 \quad x \in(0, L),
$$

and with boundary conditions at $x=0$ and $x=L$ of the following form:

$$
\left\{\begin{array}{l}
\left.u_{1}\right|_{x=L}=\left.u_{2}\right|_{x=L}=\left.v\right|_{x=L}=\left.\frac{\partial v}{\partial x}\right|_{x=L}=0 \quad t \in(0, T) \\
\left.a_{1} \frac{\partial u_{1}}{\partial x}\right|_{x=0}=f_{1}(t),\left.\quad a_{2} \frac{\partial u_{2}}{\partial x}\right|_{x=0}=f_{2}(t) \quad t \in(0, T) \\
\left.j \frac{\partial^{2} v}{\partial x^{2}}\right|_{x=0}=f_{3}(t) \quad t \in(0, T) \\
\left.\left(-\frac{\partial}{\partial x}\left(j \frac{\partial^{2} v}{\partial x^{2}}\right)+k\left(u_{2}-u_{1}+\frac{\partial v}{\partial x} e_{s}\right) e_{s}+k e_{c}^{2} \frac{\partial v}{\partial x}\right)\right|_{x=0}=f_{4}(t) \quad t \in(0, T)
\end{array}\right.
$$

Hereinafter, the quantities relative to the concrete beam and the steel beam will be denoted by indices $i=1,2$, respectively. $u_{i}(x, t)$ are the axial displacements and $v(x, t)$ is the transversal displacement of a $x$ abscissa transversal section evaluated at the moment of time $t$. We often suppress the independent variables of functions. In equations (1), $j_{i} \equiv E_{i} J_{i}$ and $a_{i} \equiv E_{i} A_{i}$ are 
the flexural stiffness and the axial stiffness of the cross-section, respectively, where $A_{i}$ and $J_{i}$ are the area and the moment of inertia of the $i$ th beam transversal section and $E_{i}, E_{i}>0$, is the relative Young's modulus of the material. The quantity $j$ is defined as $j \equiv j_{1}+j_{2}$. The coefficient $k, k(x) \geq 0$ in $[0, L]$, represents the shearing stiffness for unit length of the connection. $\rho_{i}$ is the linear mass density of the $i$ th beam, $\rho_{i}>0$ and $\rho \equiv \rho_{1}+\rho_{2}$. Finally, $e_{s}$ is the half-height of the steel beam and $e_{c} \equiv e-e_{s}$, where $e$ is the distance between the axes of the two beams forming the system.

Boundary conditions (3) express the fact that the right end-section of both of the two beams is clamped, i.e. the displacements $u_{i}, v$ and the rotation $\frac{\partial v}{\partial x}$ at $x=L$ are hindered, and the left end-section is subjected to axial and transversal forces $f_{i}(t)$ and $f_{3}(t)$, respectively, and a bending moment $f_{4}(t)$, see [BMW].

In most real applications, practical and technological requirements require to use uniform beams in forming the composite system, so that one can assume in (1) constant elastic and inertial coefficients for the steel beam and the concrete beam. Moreover, these coefficients, together with the geometrical quantities $e$ and $e_{s}$, usually are a-priori determined with high degree of accuracy, see [BMW]. It follows that the main inverse problem for this class of mechanical systems consists in estimating the coefficient $k$, which contains direct information on the integrity of the connection.

A diagnostic technique based on measuring damage induced changes in a set of lower natural frequencies has been presented in $[\mathrm{DM}]$. Here, we shall adopt a different methodology. We shall try to recover the shearing stiffness coefficient $k$ from the knowledge of the dynamical Neumannto-Dirichlet operator measured at one end of the beam. As a first step, in this paper we restrict the attention on a more simplified mechanical model of composite beam, where the coupling between bending and longitudinal motions can be neglected. Dynamical experiments performed in $[\mathrm{MR}]$ showed that this approximation might be acceptable when end transversal forces and the end bending moment are absent, and longitudinal forces only are applied at the left end of the beam. In this case, by formally taking $v=0$ in the previous model, the system (1) simplifies into a two-velocity dynamical system described by the equations

$$
\left\{\begin{array}{l}
a_{1} \frac{\partial^{2} u_{1}}{\partial x^{2}}+k\left(u_{2}-u_{1}\right)=\rho_{1} \frac{\partial^{2} u_{1}}{\partial t^{2}} \quad(x, t) \in(0, L) \times(0, T), \\
a_{2} \frac{\partial^{2} u_{2}}{\partial x^{2}}-k\left(u_{2}-u_{1}\right)=\rho_{2} \frac{\partial^{2} u_{2}}{\partial t^{2}} \quad(x, t) \in(0, L) \times(0, T), \\
\left.u_{1}\right|_{t=0}=\left.u_{2}\right|_{t=0}=0 \quad x \in(0, L), \\
\left.u_{1}\right|_{x=L}=\left.u_{2}\right|_{x=L}=0 \quad t \in(0, T), \\
\left.a_{1} \frac{\partial u_{1}}{\partial x}\right|_{x=0}=f_{1}(t),\left.\quad a_{2} \frac{\partial u_{2}}{\partial x}\right|_{x=0}=f_{2}(t) \quad t \in(0, T) .
\end{array}\right.
$$

Few results are available for inverse problems on two-velocity dynamical systems as that described in (4). The main feature of such systems is that they include waves of two types propagating with different velocities and interacting one with each other through the interval $[0, L]$.

Let us denote by $R^{T}\left(f_{1}(t), f_{2}(t)\right)=\left(u_{1}(0, t), u_{2}(0, t)\right)$ the response operator at $x=0$ for the dynamical system (4) due to an input of the form $\left(f_{1}(t), f_{2}(t)\right)$ applied at $x=0, t \in(0, T)$. In [BA] the authors studied a two-velocity dynamical system similar to (4) with a coupling on the zero order term given by a general $2 \times 2$ matrix. By using arguments related to the Boundary Control Method, they proved that the response function of the system $R^{T}$, evaluated at $x=0$ 
for some finite time $T$, uniquely determines the unknown matrix in a small interval near $x=0$, e.g. in $[0, c], 0<c<L$, where $c$ is sufficiently small, see [BA, Theorem 1] for a more precise statement.

In our case, the coupling on the zero order term involves a $2 \times 2$ matrix which has a particular

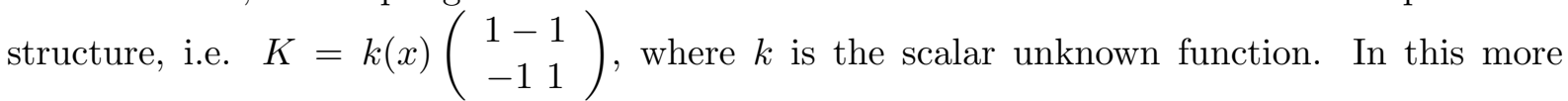
simple context and assuming $k \in C^{2}([0, L])$, we shall prove that the coefficient $k$ is uniquely determined in the whole interval $[0, L]$ from the measurement of $R^{\infty}(\delta(t), 0)$ and $R^{\infty}(0, \delta(t))$, and we shall give a reconstruction procedure to identify $k$, see Theorem 1 below. Our strategy of reconstruction is based on two main steps. After transforming (4) to an equivalent first order system, we use the progressive waves approach (see [Bla] and [Rom]) to reduce the local reconstruction of $k$ to the resolution of a system of Volterra nonlinear integral equations. An iterative use of a layer stripping technique (see [Si]) enables us to determine, step-by-step, the coefficient $k$ in the whole interval $[0, L]$.

Let us comment the amount of data needed for the reconstruction of $k$. Knowing the response operator $R^{\infty}$ is non realistic in practical situations, because the response of the system can be experimentally acquired in a finite interval of time only. Actually, since we are dealing with a one-dimensional wave propagation problem on an interval of finite length, it is enough to know the response operator $R^{T}$ for a sufficiently large but finite time $T^{*}$. This is justified by the fact that these dynamical Neumann-to-Dirichlet maps known for $T>T^{*}$ can be continued for every time $T>0$, see $[\mathrm{BA}]$ and $[\mathrm{KKL}]$. The value $T^{*}$ is the time necessary for the longitudinal waves to travel along the beams, from the left end to the right, and to reach the right boundary, e.g. $T^{*}=\max \left\{\frac{L}{\sqrt{\frac{a_{1}}{\rho_{1}}}}, \frac{L}{\sqrt{\frac{a_{2}}{\rho_{2}}}}\right\}$.

Concerning the kind of data we have considered, it is worth noticing that our set of input and output measurements corresponds to the measurement of the so-called impulsive response function at the left end of the beam, or, passing to the frequency domain, to the frequency response function at the same point. From the mechanical point of view, the experiments needed for the measurement of these quantities are easy to carry out: the impulsive force $(\delta(t), 0)$, for example, can be introduced by exciting longitudinally the first beam by means of an instrumented hammer, while the longitudinal response $\left(u_{1}(0, t), u_{2}(0, t)\right)$ can be acquired with a pair of piezoelectric accelerometers placed at the end $x=0$ of both of the two beams, see [E] for a general presentation of experimental modal analysis techniques and [MR] for a comprehensive series of dynamic tests on steel-concrete composite beams.

Finally, it is worth noticing that there are situations important in practice in which, besides the coefficient $k$, also the parameters $a_{1}$ and $a_{2}$ have to be determined, see [BMW] and [DM]. For these cases, we shall prove a global uniqueness result in the interval $[0, L]$ for the quantities $\left(k ; \rho_{1}, \rho_{2} ; a_{1}, a_{2}\right)$ from the knowledge of the response operator $R^{\infty}$, when either $\left(\rho_{1}, \rho_{2}\right)$ or $\left(a_{1}, a_{2}\right)$ are known and constant, see Theorem 2 for a precise statement and Theorem 3 for an extension to the case of piecewise constant coefficients. This result follows by the global reconstruction result for the coefficient $k$ and by using some recent results on inverse problems for dynamical two-velocities systems proved in $[\mathrm{BA}]$ and $[\mathrm{Si}]$.

The plan of the paper is as follows. In Section 2 we state our main results, Theorem 1, 
Theorem 2 and Theorem 3. In Section 3 we state some auxiliary propositions concerning certain properties of the response operator (Propositions 3.1,3.2 and 3.3), a reduction of the system (4) to a first order system (Proposition 3.4), the local reconstruction of $k$ (Proposition 3.5) and the local determination of the frequency response function (Proposition 3.6). At the end of the same Section we give the proof of Theorem 1, Theorem 2 and a sketched proof of Theorem 3. Section 4 contains a proof of the auxiliary propositions. Some concluding remarks are collected in Section 5.

\section{The main result}

Let us first introduce some notation which will be useful in what follows.

A 2-dimensional column vector $b=\left(\begin{array}{l}b_{1} \\ b_{2}\end{array}\right)$ will be simply denoted by $\left(b_{1}, b_{2}\right)$ when it used in the text.

We rewrite the two-velocity dynamical system (4) governing the small longitudinal vibrations $u=\left(u_{1}(x, t), u_{2}(x, t)\right)$ of a connected beam in the following form:

$$
\left\{\begin{array}{l}
R u_{, t t}-A u_{, x x}=-K(x) u \quad(x, t) \in(0, L) \times(0, T), \\
\left.u\right|_{t=0}=0,\left.\quad u_{, t}\right|_{t=0}=0 \quad x \in(0, L) \\
\left.A u_{, x}\right|_{x=0}=\left(\begin{array}{c}
f_{1}(t) \\
f_{2}(t)
\end{array}\right) \quad t \in(0, T) \\
\left.u\right|_{x=L}=0 \quad t \in(0, T)
\end{array}\right.
$$

where

$$
R=\left(\begin{array}{cc}
\rho_{1} & 0 \\
0 & \rho_{2}
\end{array}\right), \quad A=\left(\begin{array}{cc}
a_{1} & 0 \\
0 & a_{2}
\end{array}\right)
$$

are constant matrices, $\rho_{i}>0$ and $a_{i}>0, i=1,2$, and

$$
K(x)=k(x)\left(\begin{array}{cc}
1 & -1 \\
-1 & 1
\end{array}\right)
$$

where $k \in C^{2}([0, L]), k(x) \geq 0$ in $[0, L]$.

The quantity $\sqrt{\frac{a_{i}}{\rho_{i}}}$ represents the velocity of the longitudinal elastic wave propagating through the $i$ th beam, $i=1,2$. We set

$$
\lambda_{i}=\sqrt{\frac{\rho_{i}}{a_{i}}}, \quad i=1,2
$$

and for definiteness we shall assume

$$
\lambda_{1}<\lambda_{2} .
$$

Given an input $f(t)=\left(f_{1}(t), f_{2}(t)\right) \in\left(L^{2}(0, T)\right)^{2}$ applied at $x=0, t \in(0, T)$ and $T>0$, we shall denote by

$$
R^{T}(f(t))=\left(\begin{array}{l}
u_{1}(0, t) \\
u_{2}(0, t)
\end{array}\right) \quad t \in(0, T)
$$


the response operator at $x=0$ (or the dynamical Neumann-to-Dirichlet map evaluated at $x=0$ ) for the dynamic system (5) where $u:=\left(u_{1}(x, t), u_{2}(x, t)\right)$ is the solution of (5). The definition of $R^{T}$ will be justified in Proposition 3.1.

Let $\delta(t)$ denote the one-dimensional Dirac measure with support at $t$. We shall denote by $\epsilon$ the Heaviside function on $\mathbb{R}$.

The main result of this paper concerns, roughly speaking, the unique reconstruction of the coefficient $k$ in the dynamical system (5) from the knowledge of its response operator (9). More precisely, we prove the following global reconstruction result.

Theorem 1 Let $k \in C^{2}([0, L]), k(x) \geq 0$ in $[0, L]$. Let the response operator $R^{T}(f(t))$ of the dynamic system (5) be given in $(0, T)$, $T$ finite and $T>\lambda_{2} L$, for inputs $f(t)=(\delta(t), 0)$ and $f(t)=(0, \delta(t))$. For every $c$ in $(0, L)$, there is a reconstruction procedure to determine $k$ in the whole interval $[0, c]$.

As we said in the introduction, the proof of this theorem is of layer stripping type. We will prove that for every $c$ in $(0, L)$ we reconstruct $k(x)$ in $(0, c)$ in finitely many steps.

When, besides the coefficient $k$, also the densities $\rho_{i}$ or the axial stiffnesses $a_{i}$ are unknown, we have the following global uniqueness result. The proof will be given in Section 3 .

Theorem 2 Let us consider two dynamical systems (5) with coefficients $\left\{R_{1}, A_{1}, k_{1}\right\}$ and $\left\{R_{2}\right.$, $\left.A_{2}, k_{2}\right\}$, where $R_{j}, A_{j}$ are as above and $k_{j} \in C^{2}([0, L]), k_{j}(x) \geq 0$ in $[0, L], j=1,2$. Let $R_{j}^{T}(f(t))$ be given in $[0, T]$, with $T$ finite, $T>\lambda_{2} L$ and $j=1,2$, for inputs $f(t)=(\delta(t), 0)$ and $f(t)=(0, \delta(t))$.

If $R_{1}^{T}(\delta(t), 0)=R_{2}^{T}(\delta(t), 0)$ and $R_{1}^{T}(0, \delta(t))=R_{2}^{T}(0, \delta(t))$, then we have

i) $R_{1}=R_{2} \Leftrightarrow A_{1}=A_{2}$

and

ii) $k_{1}(x)=k_{2}(x)$ in $[0, L]$.

This last theorem has the following generalization.

Theorem 3 Let the assumptions of Theorem 2 be satisfied. Then, Theorem 2 continues to hold i) if $A_{j}, R_{j}$ are piecewise constant matrix valued functions

and

ii) if we replace the matrix $K(x)$ by a general $2 \times 2$ real valued, $C^{\infty}$ symmetric matrix.

A sketch of a proof for this last theorem will be given at the end of Section 3 .

\section{Proof of Theorem 1 and Theorem 2}

For the proof of Theorem 1, we shall need the following three propositions concerning some properties of the response operator $R^{T}$ for the system (5). The proofs of these propositions can be found in Section 4 .

Proposition 3.1 Let $T>0, T$ finite. The response operator $R^{T}$ of the system (5) is a bounded operator from $\left(L^{2}(0, T)\right)^{2}$ to $\left(L^{2}(0, T)\right)^{2}$. 
Let us consider the elliptic problem associated to (5):

$$
\left\{\begin{array}{l}
-A v_{, x x}+K(x) v-\omega^{2} R v=0 \quad x \in(\alpha, \beta) \subset(0, L) \\
\left.A v_{, x}\right|_{x=\alpha}=b,\left.\quad v\right|_{x=\beta}=0
\end{array}\right.
$$

where $\omega$ is a complex number, $v=\left(v_{1}(x), v_{2}(x)\right)$ and $b=\left(b_{1}, b_{2}\right) \in \mathbb{C}^{2}$. We shall denote by

$$
\Lambda_{\omega}^{(\alpha, \beta)}(b)=\left.v(x)\right|_{x=\alpha}
$$

the Neumann-to-Dirichlet operator for (10) at $x=\alpha$. When in the problem 10, the trace on $x=\beta$ is equal to $d$ different from zero, we use the notation

$$
\Lambda_{\omega}^{(\alpha, \beta)}\left(\begin{array}{c}
b \\
d
\end{array}\right) .
$$

This will be useful in the last section of this paper.

Next proposition correlates the Fourier transform of the response operator $R^{\infty}$ and the Neumannto-Dirichlet operator $\Lambda_{\omega}^{(0, L)}$.

Proposition 3.2 Let $f=\left(f_{1}(t), f_{2}(t)\right) \in\left(C_{0}^{\infty}\left(\mathbb{R}^{+}\right)\right)^{2}$ with support in $[0, T]$, for some fixed $T>0, T$ finite. We have

$$
\widehat{R^{\infty}(f)}(\omega)=\Lambda_{\omega}^{(0, L)}(\widehat{f}(\omega)),
$$

for every $\omega \in\left\{z \in \mathbb{C} \mid \Im z^{2}<0\right\} \cup\left\{z \in \mathbb{C} \mid z^{2} \in\left\{\mathbb{R} \backslash \sigma^{(0, L)}\right\}\right\}$, where $\sigma^{(0, L)}$ is the set of the eigenvalues $\mu$ of the following problem

$$
\left\{\begin{array}{l}
-A v_{, x x}+K(x) v-\mu R v=0 \quad x \in(0, L), \\
\left.A v_{, x}\right|_{x=0}=0, \\
\left.v\right|_{x=L}=0 .
\end{array}\right.
$$

Relation (12) can also be expressed in the following form:

$$
\widehat{R^{\infty}(f)}(\omega)=\left(\begin{array}{cc}
\Lambda_{\omega}^{1,1} & \Lambda_{\omega}^{1,2} \\
\Lambda_{\omega}^{2,1} & \Lambda_{\omega}^{2,2}
\end{array}\right)^{(0, L)}\left(\begin{array}{c}
\widehat{f}_{1}(\omega) \\
\widehat{f}_{2}(\omega)
\end{array}\right)
$$

where the $2 \times 2$ complex valued matrix $H(\omega)=\left(\begin{array}{cc}\Lambda_{\omega}^{1,1} & \Lambda_{\omega}^{1,2} \\ \Lambda_{\omega}^{2,1} & \Lambda_{\omega}^{2,2}\end{array}\right)^{(0, L)}$ is the so-called frequency response function (frf), relating the Fourier transform of the input $\left(f_{1}(t), f_{2}(t)\right)$ and that of the output $\left(u_{1}(0, t), u_{2}(0, t)\right)$. The first and the second column of $H(\omega)$ can be obtained by taking as inputs $\left(f_{1}(t), 0\right)$ and $\left(0, f_{2}(t)\right)$, respectively. Therefore, Proposition 3.2 gives a link between the dynamical response operator and the frequency response function for the longitudinal motions of the connected beam. For $\omega$ fixed, $H(\omega)$ is the matrix defining the map $\Lambda_{\omega}^{(0, L)}$.

The following proposition states that the response operator (9) for an impulsive input can be evaluated as limit of the first time-derivative (in distributional sense) of the response operator for regular functions. 
Proposition 3.3 For any sequence $\left\{f_{n}\right\}_{n \in \mathbb{N}} \subset C_{0}^{\infty}(0, T)$ such that $f_{n} \rightarrow \epsilon(t)$ in $L^{2}(0, T)$, we have

$$
R^{T}\left(\begin{array}{c}
\delta(t) \\
0
\end{array}\right)=\frac{d}{d t}\left(\lim _{n \rightarrow+\infty} R^{T}\left(\begin{array}{c}
f_{n}(t) \\
0
\end{array}\right)\right)
$$

where $\epsilon(t)$ is the Heaviside function.

The equality (15) is given in the distribution sense. We will see in the proof of this proposition that when taking $T<\lambda_{1} L$, we have the pointwise equality.

By Proposition 3.3 and the Duhamel principle one can prove that knowing the response operator $R^{T}(f)$ for every $f \in\left(L^{2}(0, T)\right)^{2}$ is equivalent to know the response operator for impulsive inputs, i.e. $R^{T}(\delta(t), 0)$ and $R^{T}(0, \delta(t))$. It is worth noticing that $R^{T}(\delta(t), 0)$ represents just the data we shall use to determine locally the coefficient $k$, see Proposition 3.5.

The local determination of the coefficient $k$ is based on two steps. In the first one, the two-bytwo second order system (5) is transformed to a first order system, see Proposition 3.4; in the second one, we shall use the progressive waves approach (see [Rom]) to reduce the reconstruction problem for $k$ to a system of nonlinear Volterra integral equations, which will be solved by successive approximations, see Proposition 3.5.

Proposition 3.4 Let $u(x, t)=\left(u_{1}(x, t), u_{2}(x, t)\right)$ be the solution of the initial-boundary value problem

$$
\left\{\begin{array}{l}
R u_{, t t}-A u_{, x x}=-K(x) u \quad(x, t) \in(0, L) \times(0, T), \\
\left.u\right|_{t=0}=0,\left.\quad u_{, t}\right|_{t=0}=0 \quad x \in(0, L) \\
\left.A u_{, x}\right|_{x=0}=\left(\begin{array}{c}
\delta(t) \\
0
\end{array}\right) \quad t \in(0, T) \\
\left.u\right|_{x=L}=0 \quad t \in(0, T)
\end{array}\right.
$$

where the matrices $R, A, K$ are defined in (6), (7).

The function $\psi=\left(\psi_{1}, \psi_{2}, \psi_{3}, \psi_{4}\right)$ defined by

$$
\left\{\begin{array}{l}
\psi_{1}=u_{1}, \\
\psi_{2}=u_{2} \\
\psi_{3}=u_{1, x}+\lambda_{1} u_{1, t}, \\
\psi_{4}=u_{2, x}+\lambda_{2} u_{2, t}
\end{array}\right.
$$


where $\lambda_{i}=\sqrt{\frac{\rho_{i}}{a_{i}}}, i=1,2$, with $\lambda_{1}<\lambda_{2}$ by assumption, satisfies the following first order problem:

$$
\left\{\begin{array}{l}
\psi_{, x}+\left(\begin{array}{cc}
\Lambda & 0 \\
0 & -\Lambda
\end{array}\right) \psi_{, t}=Q(x) \psi \quad(x, t) \in(0, L) \times(0, T) \\
\left.\psi\right|_{t=0}=0 \quad x \in(0, L) \\
\left.A\left(\begin{array}{c}
\psi_{1, x} \\
\psi_{2, x}
\end{array}\right)\right|_{x=0}=\left(\begin{array}{c}
\delta(t) \\
0
\end{array}\right) \quad t \in(0, T), \\
\left.\psi_{3}\right|_{x=0}=\frac{1}{a_{1}}+\lambda_{1} g_{1, t}(t) \quad t \in(0, T), \\
\left.\psi_{4}\right|_{x=0}=\lambda_{2} g_{2, t}(t) \quad t \in(0, T) \\
\left.\psi\right|_{x=L}=\left(\begin{array}{c}
0 \\
0 \\
\left.u_{1, x}\right|_{x=L} \\
\left.u_{2, x}\right|_{x=L}
\end{array}\right) t \in(0, T)
\end{array}\right.
$$

where $\Lambda=\left(\begin{array}{cc}\lambda_{1} & 0 \\ 0 & \lambda_{2}\end{array}\right), Q=\left(\begin{array}{cc}0 & I \\ \Lambda^{2} B & 0\end{array}\right), B=\left(\begin{array}{cc}q_{1} & -q_{1} \\ q_{2} & -q_{2}\end{array}\right), q_{1}(x)=\frac{k(x)}{\rho_{1}}, q_{2}(x)=-\frac{k(x)}{\rho_{2}}$, and $g_{i}(t)=\left.\psi_{i}\right|_{x=0}, i=1,2$.

Proposition 3.5 Given the response operator $R^{T}(\delta(t), 0)$ of the problem (5), $T<\lambda_{1} L$, there exists a sufficiently small $c, c \in(0, L)$ and $c$ depending on $R^{T}\left(f_{1} \delta(t), 0\right)$, such that the coefficient $k$ is uniquely determined in $(0, c)$ and a reconstruction procedure is given.

Remark 3.1 In Proposition 3.5 we take $T<\lambda_{1} L$. Hence, from the finite velocity of propagation of waves, the wave propagating from $x=0$ will not reach the other side at $x=L$ and, as a consequence, it will be no reflected within the interval of time $(0, T)$. This fact plays an important role in the proof of Proposition 3.5.

The following proposition enables us to determine the frf (14) corresponding to our problem (5) replacing the interval $(0, L)$ by $(c, L)$, where $c>0$ is the known length given in Proposition 3.5. The frf for the interval $(c, L)$ is obtained in a constructive way by solving a two-by-two linear system, whose matrix is given explicitly by the known frequency matrix for the whole interval $(0, L)$ and the values of $k$ on $(0, c)$.

Proposition 3.6 Let $\omega$ be a fixed number belonging to the set $\left\{z \in \mathbb{C} \mid \Im z^{2}<0\right\} \cup\left\{z \in \mathbb{C} \mid z^{2} \in\right.$ $\left.\left\{\mathbb{R} \backslash \sigma^{(0, L)}\right\}\right\}$, where $\sigma^{(0, L)}$ is the set of the eigenvalues of the problem (13), and let $c \in(0, L)$. The Neumann-to-Dirichlet operator $\Lambda_{\omega}^{(c, L)}$ of (5) can be uniquely reconstructed from the knowledge of the Neumann-to-Dirichlet operator $\Lambda_{\omega}^{(0, L)}$ of (5) and the restriction of $k$ on the subinterval $(0, c)$.

We can now give the proof of Theorem 1 .

Proof of Theorem 1. The proof is of layer stripping type and it is based on two main steps. In the first one, we reconstruct the coefficient $k$ in the subinterval $(0, c)$ of the domain 
$(0, L)$, where $0<c<L$ and the length $c$ is estimated in terms of the response operator $R^{T}$ evaluated at $x=0$. In the second step, we show how to reconstruct $k$ in any subinterval of $(0, L)$ in a finite number of steps.

By taking $T<\lambda_{1} L$, Proposition 3.5 gives a reconstruction procedure to determine $k$ in $(0, c)$, where $c$ is sufficiently small, $0<c<L$, and depends on $R^{T}(\delta(t), 0)$.

By the Duhamel principle, knowing the response operator $R^{T}(\delta(t), 0)$ and $R^{T}(0, \delta(t))$ is equivalent to know the response operator $R^{T}(f)$ evaluated on a class of $\left(L^{2}(0, T)\right)^{2}$ inputs. Moreover, since we are working on an interval of finite length $L$, the knowledge of $R^{T}(f)$, $f \in\left(C_{0}^{\infty}(0, T)\right)^{2}$, for $T>\lambda_{2} L$ is enough to determine $R^{T}(f)$ for every $T>0$, that is $R^{\infty}(f)$ (see $[\mathrm{KKL}])$. In $[\mathrm{KKL}]$, the authors used the unique continuation property for hyperbolic equations to prove the continuation of $R^{T}$ in the multidimensional case. Since we are in the one dimensional case, the use of this unique continuation property for hyperbolic equations is replaced by the domain of influence for the one dimensional Cauchy problem. This is given by changing the roles of the time and the space variables. It follows that we can determine $\Lambda_{\omega}^{(0, L)}(\widehat{f}(\omega))$ by Proposition 3.2 and, in turn, $\Lambda_{\omega}^{(c, L)}(\widehat{f}(\omega))$ by Proposition 3.6 on regular inputs $f$.

From the knowledge of the frf $\Lambda_{\omega}^{(c, L)}$, by applying Proposition 3.2 and Proposition 3.3, we can construct the response operator $R^{T}(\delta(t), 0)$ and $R^{T}(0, \delta(t))$ for the corresponding dynamical problem stated in $(c, L)$. With this response operator in hand, we can go back to the first step to reconstruct the coefficient in some interval $\left(c_{1}, L\right)$, where $c<c_{1}<L$. Therefore, step by step, we can construct the coefficient $k$ in the whole interval $(0, L)$.

For the proof of Theorem 2 we shall need the following proposition.

Proposition 3.7 Let us consider the solution $u^{j}=\left(u_{1}^{j}(x, \lambda), u_{2}^{j}(x, \lambda)\right), j=1,2$, of the following problem

$$
\left\{\begin{array}{l}
-A_{j} u_{, x x}^{j}+K_{j}(x) u^{j}-\lambda R_{j} u^{j}=0 \quad x \in(0, L), \\
\left.A_{j} u_{, x}^{j}\right|_{x=0}=\left(\begin{array}{c}
f_{1} \\
f_{2}
\end{array}\right), \\
\left.u^{j}\right|_{x=L}=0,
\end{array}\right.
$$

where $A_{j}, R_{j}$ are as in (6), with $\rho_{i}$ and $a_{i}, i=1,2$, replaced by $\rho_{i}^{j}$ and $a_{i}^{j}, i=1,2$ and $j=1,2$. $K_{j}(x)$ is a $2 \times 2$ real valued symmetric matrix, $\lambda \in \mathbb{R}$ and $f_{i} \in \mathbb{R}, i=1,2$. Here, we set $\omega^{2} \equiv \lambda$. $\Lambda_{\omega}^{j} \equiv \Lambda_{\omega}^{(0, L) j}, j=1,2$, are the Neumann-to-Dirichlet operators associated to problem (19), while $\sigma_{j}^{(0, L)}$ are the sets of eigenvalues of problem (19).

We have:

1) $\left\|u^{j}(\cdot, \lambda)\right\|_{\left(L^{2}(0, L)\right)^{2}}$ tends to zero as $\lambda$ tends to $-\infty, j=1,2$.

2) If $f_{1} \neq 0$ (respectively $\left.f_{2} \neq 0\right)$, then $\left\|\left(u_{1}^{j}\right)^{\prime}(\cdot, \lambda)\right\|_{L^{2}(0, L)} \quad$ (respectively $\left.\left\|\left(u_{2}^{j}\right)^{\prime}(\cdot, \lambda)\right\|_{L^{2}(0, L)}\right)$ tends to infinity as $\lambda$ tends to $-\infty, j=1,2$.

3) If $f_{1}=0$ (respectively $\left.f_{2}=0\right)$, then both $\left\|\left(u_{1}^{j}\right)^{\prime}(\cdot, \lambda)\right\|_{L^{2}(0, \epsilon)}$ and $\lambda\left\|u_{1}^{j}(\cdot, \lambda)\right\|_{L^{2}(0, \epsilon)}^{2}$ (respectively both $\left\|\left(u_{2}^{j}\right)^{\prime}(\cdot, \lambda)\right\|_{L^{2}(0, \epsilon)}$ and $\left.\lambda\left\|u_{2}^{j}(\cdot, \lambda)\right\|_{L^{2}(0, \epsilon)}^{2}\right)$ tend to zero for any $\epsilon<L, j=1,2$, as $\lambda$ 
tends to $-\infty$.

4) If $\Lambda_{\omega}^{1}=\Lambda_{\omega}^{2}$ for $\omega^{2}$ in $\mathbb{R}_{-}$, then for a given $f=\left(f_{1}, f_{2}\right)$ in $\mathbb{R}^{2}$ we have:

$$
\int_{0}^{L}\left(A_{2}-A_{1}\right) u^{2^{\prime}}(\cdot, \lambda) \cdot u^{2^{\prime}}(\cdot, \lambda)+\left(\left(K_{2}-K_{1}\right)-\lambda\left(R_{2}-R_{1}\right)\right) u^{2}(\cdot, \lambda) \cdot u^{2}(\cdot, \lambda) d x \leq 0
$$

and

$$
\int_{0}^{L}\left(A_{1}-A_{2}\right) u^{1^{\prime}}(\cdot, \lambda) \cdot u^{1^{\prime}}(\cdot, \lambda)+\left(\left(K_{1}-K_{2}\right)-\lambda\left(R_{1}-R_{2}\right)\right) u^{1}(\cdot, \lambda) \cdot u^{1}(\cdot, \lambda) d x \leq 0
$$

for all $\lambda<0$.

We refer to [Si, Lemma 2.2 and Lemma 5.1] for a proof of this proposition.

We are now in position to prove Theorem 2.

Proof of Theorem 2. Assume that $R_{1}^{T}(f)=R_{2}^{T}(f)$, for $T>\lambda_{2} L$ and for inputs $f(t)=$ $(\delta(t), 0)$ and $f(t)=(0, \delta(t))$. Hence, by the Duhamel principle, we have also $R_{1}^{T}(f)=R_{2}^{T}(f)$ for every $f \in\left(L^{2}(0, T)\right)^{2}$. Arguing as in the proof of Theorem 1, we deduce that $R_{1}^{\infty}(f)=R_{2}^{\infty}(f)$ for every $f(t) \in\left(C_{0}^{\infty}(0,+\infty)\right)^{2}$. Therefore, from Proposition 3.2, we have

$$
\Lambda_{\omega}^{1}=\Lambda_{\omega}^{2}
$$

for every $\omega \in\left\{z \in \mathbb{C} \mid \Im z^{2}<0\right\} \cup\left\{z \in \mathbb{C} \mid z^{2} \in\left\{\mathbb{R} \backslash\left(\sigma_{1}^{(0, L)} \cup \sigma_{2}^{(0, L)}\right)\right\}\right\}$. Since $\Lambda_{\omega}^{j}$ is a meromorphic function with respect to $\omega$ and has $\sigma_{j}^{(0, L)}$ as the set of poles, $j=1,2$, we deduce that $\Lambda_{\omega}^{1}=\Lambda_{\omega}^{2}$ for every $\omega \in \mathbb{C} \backslash\left(\sigma_{1}^{(0, L)} \cup \sigma_{2}^{(0, L)}\right)$. Moreover, using equality (22), we deduce that $\sigma_{1}^{(0, L)}=\sigma_{2}^{(0, L)}$.

Let us recall Alessandrini's identity for the system (19):

$$
\begin{gathered}
\int_{0}^{L}\left(A_{2}-A_{1}\right) u^{2^{\prime}}(\cdot, \lambda) \cdot u^{1^{\prime}}(\cdot, \lambda)+\left(\left(K_{2}-K_{1}\right)-\lambda\left(R_{2}-R_{1}\right)\right) u^{2}(\cdot, \lambda) \cdot u^{1}(\cdot, \lambda) d x= \\
=\left(\Lambda_{\omega}^{2}(f)-\Lambda_{\omega}^{1}(f)\right) \cdot g
\end{gathered}
$$

for every $H^{1}(0, L)$-solution of $(19)$, where $f=\left(f_{1}, f_{2}\right)$ and $g=\left(g_{1}, g_{2}\right)$ are the traces on $x=0$ of $A_{2} u^{2}(\cdot, \lambda)^{\prime}$ and $A_{1} u^{1}(\cdot, \lambda)^{\prime}$ respectively.

Now, by (22) and (23) we deduce that the left hand side in (23) is zero. Let $\Lambda_{\omega}^{D, j}$ and $\sigma_{j, D}^{(0, L)}$ be, respectively, the associated Dirichlet-to-Neumann operators and the set of eigenvalues of the problem (19), replacing the Neumann condition by the Dirichlet one at $x=0$. Using Alessandrini's identity associated to this last problem, we deduce that

$$
\Lambda_{\omega}^{D, 1}=\Lambda_{\omega}^{D, 2},
$$

for every $\omega \in \mathbb{C} \backslash\left(\sigma_{1, D}^{(0, L)} \cup \sigma_{2, D}^{(0, L)}\right)$, (cf. [Si]). 
From the equality $\Lambda_{\omega}^{D, 1}=\Lambda_{\omega}^{D, 2}$ for every $\omega^{2}<0$, we shall prove that $A_{1}=A_{2}$ (or $R_{1}=R_{2}$ ). Suppose, for instance, that $R_{1}=R_{2}$. Then we shall prove that $A_{1}=A_{2}$. The other implication can be proven similarly. Assume that $a_{1}^{1}-a_{1}^{2}>0$. From (21) we have

$$
\begin{gathered}
0 \geq\left(a_{1}^{1}-a_{1}^{2}\right) \int_{0}^{L}\left(u_{1}^{1^{\prime}}(\cdot, \lambda)\right)^{2} d x+\int_{0}^{L}\left(K_{1}-K_{2}\right) u^{1}(\cdot, \lambda) \cdot u^{1}(\cdot, \lambda) d x+ \\
+\left(a_{2}^{1}-a_{2}^{2}\right) \int_{0}^{L}\left(u_{2}^{1^{\prime}}(\cdot, \lambda)\right)^{2} d x .
\end{gathered}
$$

If we choose $f_{2}=0$, then from Proposition 3.7, property 3$), \int_{0}^{L}\left(u_{2}^{1^{\prime}}(\cdot, \lambda)\right)^{2} d x$ tends to zero as $\lambda$ tends to $-\infty$. From property 1$)$ of the same proposition, we have that $\int_{0}^{L}\left(K_{1}-K_{2}\right) u^{1}(\cdot, \lambda)$. $u^{1}(\cdot, \lambda) d x$ tends to zero as $\lambda$ tends to $-\infty$. Moreover, by taking $f_{1} \neq 0$ in Proposition 3.7, property 2) implies that $\int_{0}^{L}\left(u_{1}^{1^{\prime}}(\cdot, \lambda)\right)^{2} d x$ tends to $+\infty$ as $\lambda$ tends to $-\infty$. This fact contradicts (25). Hence, by repeating the argument using equation (20), we deduce that $a_{1}^{1}=a_{1}^{2}$. Arguing in a similar way, we can prove that $a_{2}^{1}=a_{2}^{2}$ and, therefore, $A_{1}=A_{2}$. Finally, by applying Theorem 1, we prove that $K_{1}=K_{2}$.

Sketch of the proof for Theorem 3.

Since Proposition 3.7 is valid also in this case, see [Si], we can use it to identify one of the matrices $A_{j}$ or $R_{j}$. After that, we can use the following uniqueness result in the small space interval proven in $[\mathrm{BA}]$ :

Theorem. ([BA])

Consider the two velocity system:

$$
\left\{\begin{array}{l}
R u_{, t t}-A u_{, x x}+Q(x) u=0, \quad x>0, \quad t>0 \\
\left.u\right|_{t=0}=0 \quad x>0 \\
\left.u\right|_{x=0}=f \quad t>0
\end{array}\right.
$$

where $R$ and $A$ are $2 \times 2$, diagonal and constant matrices as in (6), while $Q$ is a $C_{0}^{\infty}(0, \infty)$, $2 \times 2$ real valued symmetric matrix. If we know the response operator $R^{T}$ of the system (26) for a fixed $T>0$, then there exists $c>0$ "small enough", $c$ depending on $R^{T}$, such that $Q$ is uniquely determined in $(0, c)$.

In [BA], this result is proven for a more general self-adjoint system. In our case, we are considering Neumann boundary conditions at $x=0$. Using the Fourier transform and Alessandrini's identity as in the proof of Theorem 2, we can transform this Neumann condition to the Dirichlet one. Therefore, the above Theorem can be applied to our case, where an interval of finite length instead of a semi-infinite interval is considered. This is justified by the finiteness of the velocity of propagation of waves. Therefore, it is enough to take $T$ small enough to prove uniqueness in the small. Moreover, by using the Fourier transform (Proposition 3.3) and Proposition 3.6, we can deduce the response operator replacing the interval $(0, L)$ by $(c, L)$. Arguing step by step, we can finally deduce the uniqueness in the whole interval $(0, L)$. 


\section{Proof of the auxiliary propositions}

Proof of Proposition 3.1. Without loss of generality we can assume $L=1$.

We first show that the response operator is well defined for "regular" inputs.

Let $f \in\left(H^{1}(0, T)\right)^{2}$, with $f=\left(f_{1}(t), f_{2}(t)\right)$ such that $f_{1}(0)=f_{2}(0)=0$. The solution $\tilde{u}=\left(\tilde{u}_{1}, \tilde{u}_{2}\right)$ of the following problem

$$
\left\{\begin{array}{l}
R \tilde{u}_{, t t}-A \tilde{u}_{, x x}=0 \quad(x, t) \in(0,1) \times(0, T) \\
\left.\tilde{u}\right|_{t=0}=0,\left.\quad \tilde{u}_{, t}\right|_{t=0}=0 \quad x \in(0,1) \\
\left.A \tilde{u}_{, x}\right|_{x=0}=f \quad t \in(0, T) \\
\left.\tilde{u}\right|_{x=1}=0 \quad t \in(0, T)
\end{array}\right.
$$

is given by

$$
\tilde{u}_{i}(x, t)=-\sum_{j=0}^{\infty} \frac{(-1)^{j}}{a_{i} \lambda_{i}}\left[\left(h\left(t-\lambda_{i} x-2 j \lambda_{i}\right)-h\left(t+\lambda_{i} x-2(j+1) \lambda_{i}\right)\right)\right], \quad i=1,2,
$$

where $h(t)=\int_{0}^{t} f(s) d s$.

The series on the right hand side of (28) has a finite number of terms for every fixed $T$, hence $\tilde{u}$ is in $\left(H^{2}((0,1) \times(0, T))\right)^{2}$.

Now, the solution $u^{f}$ of the problem

$$
\left\{\begin{array}{l}
R u_{, t t}^{f}-A u_{, x x}^{f}=-K(x) u^{f} \quad(x, t) \in(0,1) \times(0, T), \\
\left.u^{f}\right|_{t=0}=0,\left.\quad u_{, t}^{f}\right|_{t=0}=0 \quad x \in(0,1), \\
\left.A u_{, x}^{f}\right|_{x=0}=f \quad t \in(0, T), \\
\left.u^{f}\right|_{x=1}=0 \quad t \in(0, T),
\end{array}\right.
$$

can be written as $u^{f}=\tilde{u}+v$, where $v$ satisfies

$$
\left\{\begin{array}{l}
R v_{, t t}-A v_{, x x}+K(x) v=-K(x) \tilde{u} \quad(x, t) \in(0,1) \times(0, T), \\
\left.v\right|_{t=0}=0,\left.\quad v_{, t}\right|_{t=0}=0, \quad x \in(0,1) \\
\left.A v_{, x}\right|_{x=0}=0 \quad t \in(0, T) \\
\left.v\right|_{x=1}=0 \quad x \in(0,1)
\end{array}\right.
$$

Since the right hand side of $(30)_{1}$ is in $\left(L^{2}((0,1) \times(0, T))\right)^{2}$, then problem (30) has a unique weak solution $v \in\left[H^{1}((0,1) \times(0, T))\right]^{2}($ see $[\mathrm{Lad}]$ or $[\mathrm{W}])$. Hence $u^{f}$ is in $\left(H^{1}((0,1) \times(0, T))\right)^{2}$ and then $R^{T}(f)=\left.u\right|_{x=0} \in H^{\frac{1}{2}}(0, T)$ is well defined. This means that we can define the response operator $R^{T}$ on $D\left(R^{T}\right)=\left\{f \in\left(H^{1}(0, T)\right)^{2} \mid f(0)=0\right\}$.

The goal of the following part is to extend the domain of definition of this response operator to $\left(L^{2}(0, T)\right)^{2}$.

Let us consider the following problem:

$$
\left\{\begin{array}{l}
R u_{, t t}^{\delta(1)}-A u_{, x x}^{\delta(1)}=-K(x) u^{\delta(1)} \quad(x, t) \in(0,1) \times(0, T) \\
\left.u^{\delta(1)}\right|_{t=0}=0,\left.\quad u_{, t}^{\delta(1)}\right|_{t=0}=0 \quad x \in(0,1) \\
\left.A u_{, x}^{\delta(1)}\right|_{x=0}=\left(\begin{array}{c}
\delta(t) \\
0
\end{array}\right) \quad t \in(0, T) \\
\left.u^{\delta(1)}\right|_{x=1}=0 \quad t \in(0, T)
\end{array}\right.
$$


We look for the solution of (31) as a sum of a singular and a regular part, namely

$$
u^{\delta(1)}=u_{s}^{\delta(1)}+u_{r}^{\delta(1)},
$$

where the singular part $u_{s}^{\delta(1)}$ satisfies the problem

$$
\left\{\begin{array}{l}
R\left(u_{s}^{\delta(1)}\right)_{, t t}-A\left(u_{s}^{\delta(1)}\right)_{, x x}=0 \quad(x, t) \in(0,1) \times(0, T), \\
\left.u_{s}^{\delta(1)}\right|_{t=0}=0,\left.\quad\left(u_{s}^{\delta(1)}\right)_{, t}\right|_{t=0}=0 \quad x \in(0,1), \\
\left.A\left(u_{s}^{\delta(1)}\right)_{, x}\right|_{x=0}=\left(\begin{array}{c}
\delta(t) \\
0
\end{array}\right) \quad t \in(0, T), \\
\left.u_{s}^{\delta(1)}\right|_{x=1}=0 \quad t \in(0, T) .
\end{array}\right.
$$

The function $u_{s}^{\delta(1)}$ is given by replacing in (28) the boundary data $g(t)$ by $(\epsilon(t), 0)$, where $\epsilon(t)$ is the Heaviside function, i.e.

$$
u_{s 1}^{\delta(1)}=-\sum_{j=0}^{\infty} \frac{(-1)^{j}}{a_{1} \lambda_{1}}\left[\left(\epsilon\left(t-\lambda_{1} x-2 j \lambda_{1}\right)-\epsilon\left(t+\lambda_{1} x-2(j+1) \lambda_{1}\right)\right)\right]
$$

and $u_{s 2}^{\delta(1)}=0$.

The regular part $u_{r}^{\delta(1)}$ satisfies the following problem:

$$
\left\{\begin{array}{l}
R\left(u_{r}^{\delta(1)}\right)_{, t t}-A\left(u_{r}^{\delta(1)}\right)_{, x x}+K(x) u_{r}^{\delta(1)}=-K(x) u_{s}^{\delta(1)} \quad(x, t) \in(0,1) \times(0, T), \\
\left.u^{\delta(1)}\right|_{t=0}=0,\left.\quad u_{, t}^{\delta(1)}\right|_{t=0}=0 \quad x \in(0,1) \\
\left.A u_{x}^{\delta(1)}\right|_{x=0}=0 \quad t \in(0, T) \\
\left.u^{\delta(1)}\right|_{x=1}=0 \quad t \in(0, T)
\end{array}\right.
$$

Since the non-homogeneous term in (34) belongs to $\left(L^{2}((0,1) \times(0, T))\right)^{2}$, the function $u_{r}^{\delta(1)}$ is in $\left(H^{1}((0,1) \times(0, T))\right)^{2}$.

By analogous considerations we define $u^{\delta(2)}$ as the solution to (31) for the boundary data $(0, \delta(t))$. We still denote by $u_{s}^{(2)}$ and $u_{r}^{(2)}$ the singular and regular parts of $u^{\delta(2)}$, respectively. Therefore, we can define the matrix solution

$$
U^{\delta}=\left(\begin{array}{ll}
u_{1}^{\delta(1)} & u_{1}^{\delta(2)} \\
u_{2}^{\delta(1)} & u_{2}^{\delta(2)}
\end{array}\right)
$$

of the problem

$$
\left\{\begin{array}{l}
R U_{, t t}^{\delta}-A U_{, x x}^{\delta}=-K(x) U^{\delta} \quad(x, t) \in(0,1) \times(0, T) \\
\left.U^{\delta}\right|_{t=0}=0,\left.\quad U_{, t}^{\delta}\right|_{t=0}=0 \quad x \in(0,1) \\
\left.A U_{, x}^{\delta}\right|_{x=0}=\left(\begin{array}{cc}
\delta(t) & 0 \\
0 & \delta(t)
\end{array}\right) \quad t \in(0, T) \\
\left.U^{\delta}\right|_{x=1}=0 \quad t \in(0, T)
\end{array}\right.
$$

and, as before, we can write

$$
U^{\delta}=U_{s}^{\delta}+U_{r}^{\delta}=\left(\begin{array}{cc}
u_{s 1}^{\delta(1)} & 0 \\
0 & u_{s 2}^{\delta(2)}
\end{array}\right)+\left(\begin{array}{cc}
u_{r 1}^{\delta(1)} & u_{r 1}^{\delta(2)} \\
u_{r 2}^{\delta(1)} & u_{r 2}^{\delta(2)}
\end{array}\right)
$$


Let us now consider an input $f(t)=\left(f_{1}(t), f_{2}(t)\right)$, with $f_{i} \in H^{1}(0, T)$ and $f_{i}(0)=0, i=1,2$. Then, in accordance with the Duhamel principle, the solution $U^{f}=\left(U_{1}^{f}, U_{2}^{f}\right)$ to $(27)$ can be written in the convolution form:

$$
U^{f}(x, t)=U^{\delta} * f(t)=U_{s}^{\delta} * f(t)+U_{r}^{\delta} * f(t) .
$$

By considering the expressions of $U_{s}^{\delta}$, assuming that $f(t-s)=0$ if $t<s$ and taking into account the finite velocity of propagation of waves, equation (36) becomes

$$
\begin{gathered}
U^{f}(x, t)=\int_{\lambda_{1} x}^{t}\left(\begin{array}{cc}
u_{s 1}^{\delta(1)}(x, s) & 0 \\
0 & u_{s 2}^{\delta(2)}(x, s)
\end{array}\right)\left(\begin{array}{c}
f_{1}(t-s) \\
f_{2}(t-s)
\end{array}\right) d s+ \\
\quad+\int_{\lambda_{1} x}^{t}\left(\begin{array}{cc}
u_{r 1}^{\delta(1)}(x, s) & u_{r 1}^{\delta(2)}(x, s) \\
u_{r 2}^{\delta(1)}(x, s) & u_{r 2}^{\delta(2)}(x, s)
\end{array}\right)\left(\begin{array}{l}
f_{1}(t-s) \\
f_{2}(t-s)
\end{array}\right) d s .
\end{gathered}
$$

Equation (37) has a meaning also when $f \in\left(L^{2}(0, T)\right)^{2}$ and, in fact, one can verify by direct calculation that the function $U^{f}(x, t)$ given in $(37)$ is the unique weak solution of the problem (27) for any given $f \in\left(L^{2}(0, T)\right)^{2}$.

From $(37), U^{f}(0, t)$ is well defined and it is given by

$$
\begin{gathered}
U^{f}(0, t)=\int_{0}^{t}\left(\begin{array}{cc}
u_{s 1}^{\delta(1)}(0, s) & 0 \\
0 & u_{s 2}^{\delta(2)}(0, s)
\end{array}\right)\left(\begin{array}{l}
f_{1}(t-s) \\
f_{2}(t-s)
\end{array}\right) d s+ \\
\quad+\int_{0}^{t}\left(\begin{array}{cc}
u_{r 1}^{\delta(1)}(0, s) & u_{r 1}^{\delta(2)}(0, s) \\
u_{r 2}^{\delta(1)}(0, s) & u_{r 2}^{\delta(2)}(0, s)
\end{array}\right)\left(\begin{array}{l}
f_{1}(t-s) \\
f_{2}(t-s)
\end{array}\right) d s
\end{gathered}
$$

In particular, for $T<\lambda_{1}$ we have

$$
\begin{aligned}
& U^{f}(0, t)=\int_{0}^{t}\left(\begin{array}{cc}
\frac{-1}{a_{1} \lambda_{1}} & 0 \\
0 & \frac{-1}{a_{2} \lambda_{2}}
\end{array}\right)\left(\begin{array}{c}
f_{1}(t-s) \\
f_{2}(t-s)
\end{array}\right) d s+ \\
& +\int_{0}^{t}\left(\begin{array}{cc}
u_{r 1}^{\delta(1)}(0, s) & u_{r 1}^{\delta(2)}(0, s) \\
u_{r 2}^{\delta(1)}(0, s) & u_{r 2}^{\delta(2)}(0, s)
\end{array}\right)\left(\begin{array}{c}
f_{1}(t-s) \\
f_{2}(t-s)
\end{array}\right) d s .
\end{aligned}
$$

Equation (39) shows that the response operator $R^{T}$ is bounded from $\left(L^{2}(0, T)\right)^{2}$ to $\left(L^{2}(0, T)\right)^{2}$, and the proof of the proposition is complete.

To prove Proposition 3.2 we need the following well known law of conservation of energy. 
Lemma 4.1 Let $u(x, t)=\left(u_{1}(x, t), u_{2}(x, t)\right)$ be the solution of $(5)$ for $f$ given as in Proposition 3.2. Let $T>0, T$ finite, as in Proposition 3.2. Then

$$
E(t)=-\int_{0}^{t}\left(\begin{array}{c}
f_{1}(s) \\
f_{2}(s)
\end{array}\right) \cdot\left(\begin{array}{c}
u_{1, s}(0, s) \\
u_{2, s}(0, s)
\end{array}\right) d s
$$

and

$$
E(t) \leq C(T)\|f\|_{\left(H^{1}\left(0, T^{*}\right)\right)^{2}}^{2}
$$

for every $t>0$, where

$$
E(t)=\frac{1}{2} \int_{0}^{L}\left(\sum_{i=1,2}\left(\rho_{i} u_{i, t}^{2}+a_{i} u_{i, x}^{2}\right)+\sum_{i, j=1,2} K_{i j}(x) u_{i} u_{j}\right) d x
$$

and the constant $C(T), C(T)>0$, depends only on $T$.

Proof of Lemma 4.1. The equality (40) follows from (5) by multiplying the equation by the time derivative of $u$ and integrating by parts.

Let us now prove the inequality (41). We recall that the solution of (5) can be written as $u=\tilde{u}+v$ as in the proof of Proposition 3.1, see equations (27)-(30). Since the function $h(t)$ appearing in (28) is the primitive of $f(t)$, then $h(t)$ is an $H^{2}$-function. Therefore, the sum in $(28)$ is finite and $\tilde{u} \in\left(H^{2}((0, L) \times(0, T))\right)^{2}$ for every finite $T>0$. From (28), we have

$$
\left\|\tilde{u}_{, t}(., .)\right\|_{\left(L^{2}((0, L) \times(0, T))\right)^{2}} \leq c(T)\|f\|_{\left(L^{2}(0, T)\right)^{2}}
$$

and

$$
\left\|\tilde{u}_{, t}(0, .)\right\|_{\left(L^{2}(0, T)\right)^{2}} \leq c(T)\|f\|_{\left(L^{2}(0, T)\right)^{2}} .
$$

Now, since the right hand side of (30) and its derivative with respect to time are in $\left(L^{2}((0,1) \times(0, T))\right)^{2}$, then $v \in\left(H^{2}((0, L) \times(0, T))\right)^{2}$, see [Lad] or [W]. Taking the time Derivative in (30), we deduce that $v_{, t}$ satisfies the problem (30) replacing $\tilde{u}$ by $\tilde{u}_{, t}$. Hence from Theorem 29.1 of $[\mathrm{W}]$ and the trace theorem, we deduce that

$$
\left\|v_{, t}(0, .)\right\|_{\left(L^{2}(0, T)\right)^{2}} \leq c^{\prime}\left\|\tilde{u}_{, t}(., .)\right\|_{\left(L^{2}((0, L) \times(0, T))\right)^{2}} \leq c(T) c^{\prime}(T)\|f\|_{\left(L^{2}(0, T)\right)^{2}} .
$$

Using the Cauchy-Schwartz inequality and the previous inequalities, we have

$$
\begin{aligned}
& E(t)=-\int_{0}^{T}\left(\begin{array}{c}
f_{1}(s) \\
f_{2}(s)
\end{array}\right) \cdot\left(\begin{array}{c}
u_{1, s}(0, s) \\
u_{2, s}(0, s)
\end{array}\right) d s \leq\|f\|_{\left(L^{2}(0, T)\right)^{2}}\left\|u_{, t}\right\|_{\left(L^{2}(0, T)\right)^{2}} \leq \\
& \leq C(T)\|f\|_{\left(L^{2}(0, T)\right)^{2}}^{2} .
\end{aligned}
$$

This ends the proof of the lemma.

Proof of Proposition 3.2. The functions $t \rightarrow u_{, x x}(x, t)$ and $t \rightarrow u_{, t t}(x, t)$ are at most exponentially growing as $e^{c t}$, where $c>0$ is a constant (see for example [KKL], p. 206). Then, 
we can take the Fourier transform with respect to time $\widehat{u(x, t)}(\omega)=\int_{\mathbb{R}} e^{-i \omega t} u(x, t) d t$ for the solution $u(x, t)$ of the problem (5) to obtain

$$
\widehat{R^{\infty}(f)}(\omega)=\Lambda_{\omega}^{(0, L)}(\widehat{f}(\omega)),
$$

for every $\omega \in\left\{z \in \mathbb{C} \mid \Im z^{2}<-c\right\}$.

From Lemma 4.1, the function $t \rightarrow u_{, x}(x, t)$, from $\mathbb{R}$ to $\left(L^{2}(0, L)\right)^{2}$, is bounded. Using Poincaré's inequality on $(0, L)$, we deduce that the function $t \rightarrow u(x, t)$ from $\mathbb{R}$ to $\left(H^{1}(0, L)\right)^{2}$ is bounded and, therefore, the function $t \rightarrow u(0, t)$ from $\mathbb{R}$ to $\mathbb{R}^{2}$ is also bounded. Hence, by applying the Fourier transform, $\widehat{R^{\infty}(f)}(\omega)$ is well defined and (42) holds in $\omega \in\left\{z \in \mathbb{C} \mid \Im z^{2}<0\right\}$.

Conversely, if we know $\widehat{R^{\infty}(f)}(\omega)$ for every $\omega \in\left\{z \in \mathbb{C} \mid \Im z^{2}<0\right\}$, then we know $\widehat{R^{\infty}(f)}(\omega-$ $i \epsilon)$ and hence $e^{-\widehat{\epsilon t} R^{\infty}}(f)$ for $\omega \in \mathbb{R}$, for some $\epsilon>0$. By the inverse Fourier transform, we deduce $e^{-\epsilon t} R^{\infty}(f)$ and hence $R^{\infty}(f)$.

Proof of Proposition 3.3. Let $\left(f_{n}\right)_{n \in \mathbb{N}} \subset C_{0}^{\infty}(0, T)$ be a sequence such that $\left.f_{n}\right|_{(0, T)} \rightarrow$ $\varepsilon$ in $L^{2}(0, T)$. Since the response operator $R^{T}$ is bounded from $\left(L^{2}(0, T)\right)^{2}$ to $\left(L^{2}(0, T)\right)^{2}$, see Proposition 3.1, we have $R^{T}\left(\begin{array}{c}f_{n} \\ 0\end{array}\right) \rightarrow R^{T}\left(\begin{array}{c}\varepsilon \\ 0\end{array}\right)$ in $\left(L^{2}(0, T)\right)^{2}$. This means we know $R^{T}\left(\begin{array}{c}\varepsilon \\ 0\end{array}\right)$ in $L^{2}$-sense, but $R^{T}\left(\begin{array}{l}\varepsilon \\ 0\end{array}\right)$ is continuous since its singular and regular parts are continuous, then it is known pointwise. Replacing $f$ by $\left(\begin{array}{c}\varepsilon \\ 0\end{array}\right)$ in $(38)$, we obtain $u^{\varepsilon}(0, t)=$ $\int_{0}^{t} u^{\delta(1)}(0, s) d s$ and therefore

$$
u^{\delta(1)}(0, t)=\frac{d}{d t}\left(u^{\varepsilon}(0, t)\right)=\frac{d}{d t}\left(\lim _{n \rightarrow \infty} R^{T}\left(\begin{array}{c}
f_{n} \\
0
\end{array}\right)\right) .
$$

If we take $T<\lambda_{1} L$, then $u^{\delta(1)}$ is continuous. Indeed, the trace on $x=0$ of the singular part of $u^{\delta(1)}$ is just $\frac{-1}{\lambda_{1} a_{1}} \epsilon(t)$, see (33). The regular part is also continuous then the last equality is true pointwise.

Proof of Proposition 3.4. The proof of this proposition follows easily by direct computation.

Proof of Proposition 3.5. By Proposition 3.4, the second order problem (5) with $f=$ $\left(\begin{array}{c}\delta(t) \\ 0\end{array}\right)$ can be reduced to the first order problem (18). We look for a solution of (18) in the form

$$
\left\{\begin{array}{l}
\psi_{1}(x, t)=-\lambda_{1}^{-1} a_{1}^{-1} \epsilon\left(t-\lambda_{1} x\right)+\psi_{01}(x, t) \\
\psi_{2}(x, t)=\psi_{02}(x, t) \\
\psi_{3}(x, t)=\psi_{03}(x, t) \\
\psi_{4}(x, t)=\psi_{04}(x, t)
\end{array}\right.
$$


where $\psi_{0}=\left(\psi_{01}, \psi_{02}, \psi_{03}, \psi_{04}\right)$ is a vector of terms smoother than the Heaviside function $\epsilon$. Then, by substituting the expression (43) of $\psi_{i}(x, t)$ into equation (18) and comparing the terms with $\epsilon$ and $\delta$, we get the following first order system for $\psi_{0}$

$$
\left\{\begin{array}{l}
\psi_{01, x}+\lambda_{1} \psi_{01, t}=\psi_{03}, \\
\psi_{02, x}+\lambda_{2} \psi_{02, t}=\psi_{04}, \\
\psi_{03, x}-\lambda_{1} \psi_{03, t}=\lambda_{1} a_{1}^{-1} q_{1}(x) \epsilon\left(t-\lambda_{1} x\right)+\lambda_{1}^{2} q_{1}(x)\left(\psi_{02}-\psi_{01}\right), \\
\psi_{04, x}-\lambda_{2} \psi_{04, t}=\lambda_{2}^{2} \lambda_{1}^{-1} a_{1}^{-1} q_{2}(x) \epsilon\left(t-\lambda_{1} x\right)+\lambda_{2}^{2} q_{2}(x)\left(\psi_{02}-\psi_{01}\right),
\end{array}\right.
$$

with initial conditions

$$
\left.\psi_{0}\right|_{t=0}=0 \quad x \in(0, L),
$$

and boundary conditions at $x=0$

$$
\left\{\begin{array}{l}
\left.\psi_{01, x}\right|_{x=0}=0 \\
\left.\psi_{02, x}\right|_{x=0}=0 \\
\left.\psi_{03}\right|_{x=0}=\lambda_{1} g_{1, t}(t) \\
\left.\psi_{04}\right|_{x=0}=\lambda_{2} g_{2, t}(t)
\end{array}\right.
$$

where $\left.g_{i}(t) \equiv \psi_{i}\right|_{x=0}$ for $t \in(0, T), i=1,2$. Since $k(x)$ is in $C^{2}[0, L]$, then the solution of (16) has the $C^{2}$ regularity outside the two characteristics, hence the trace on $x=0$ is of class $C^{2}$ and then $g_{i}(t)$ are of class $C^{2}$.

Since we are interested in solving problem (18) in a small interval of time $(0, T)$, in the following we shall choose $T$ small enough, e.g. $T<\lambda_{1} L$, so that the boundary condition at $x=L$ doesn't influence the solution we are looking for.

The solution $\psi_{0}$ of problem (44), (45), (46) is equal to zero for $t \leq \lambda_{1} x$. This is a consequence of the homogeneous initial conditions (45) and of the finite speed of propagation of waves for problem (44).

To determine $\psi_{0}$ in the remaining part of the $x-t$ plane, by integrating over the characteristics on $t \geq \lambda_{1} x$, we shall show that the solution $\psi_{0}$ of (44), (45), (46) satisfies a system of integral equations of Volterra type.

Let $P(\bar{x}, \bar{t})$, with $\bar{t}>\lambda_{1} \bar{x}$, and consider the characteristic lines through $P$ for $t \leq \bar{t}$. More precisely, when $\bar{t}>\lambda_{2} \bar{x}$, two characteristic lines with positive slope, $\lambda_{1}$ and $\lambda_{2}$, intersect the $t$ axis, where the boundary data for $\left.\psi_{03}\right|_{x=0}$ and $\left.\psi_{04}\right|_{x=0}$ is given, see (46) $)_{3,4}$; and two characteristic lines with negative slope, $-\lambda_{1}$ and $-\lambda_{2}$, intersect the straight line $t=\lambda_{1} x$, where the initial data $\psi_{0}=0$ is given. If $\lambda_{2} \bar{x} \geq \bar{t} \geq \lambda_{1} \bar{x}$, then only one characteristic line, with positive slope $\lambda_{1}$, intersects the $t$-axis, while three characteristic lines, one with positive slope $\lambda_{2}$ and two with negative slope, $-\lambda_{1},-\lambda_{2}$, intersect the straight line $t=\lambda_{1} x$.

By integrating along the characteristic lines through $P$, the following system of integral equations is obtained

$$
\left\{\begin{array}{l}
\psi_{01}(\bar{x}, \bar{t})=g_{1}\left(\bar{t}-\lambda_{1} \bar{x}\right)+\int_{0}^{\bar{x}} \psi_{03}\left(\xi, \lambda_{1}(\xi-\bar{x})+\bar{t}\right) d \xi \\
\psi_{02}(\bar{x}, \bar{t})=\widetilde{g}_{2}\left(\bar{t}-\lambda_{2} \bar{x}\right)+\int_{a}^{x} \psi_{04}\left(\xi, \lambda_{2}(\xi-\bar{x})+\bar{t}\right) d \xi \\
\psi_{03}(\bar{x}, \bar{t})=a_{1}^{-1} \lambda_{1} \int_{\frac{\lambda_{1} \bar{x}+\bar{t}}{2 \lambda_{1}}}^{\bar{x}} q_{1}(\xi) d \xi+\lambda_{1}^{2} \int_{\frac{\lambda_{1} \bar{x}+\bar{t}}{2 \lambda_{1}}}^{\bar{x}} q_{1}(\xi)\left(\psi_{02}-\psi_{01}\right)\left(\xi, \lambda_{1}(\bar{x}-\xi)+\bar{t}\right) d \xi \\
\psi_{04}(\bar{x}, \bar{t})=\lambda_{2}^{2} \lambda_{1}^{-1} a_{1}^{-1} \int_{\frac{\lambda_{2} \bar{x}+\bar{t}}{\lambda_{1}+\lambda_{2}}}^{\bar{x}} q_{2}(\xi) d \xi+\lambda_{2}^{2} \int_{\frac{\lambda_{2} \bar{x}+\bar{t}}{\lambda_{1}+\lambda_{2}}}^{\bar{x}} q_{2}(\xi)\left(\psi_{02}-\psi_{01}\right)\left(\xi, \lambda_{2}(\bar{x}-\xi)+\bar{t}\right) d \xi
\end{array}\right.
$$


where

$$
\widetilde{g}_{2}\left(\bar{t}-\lambda_{2} \bar{x}\right)=g_{2}\left(\bar{t}-\lambda_{2} \bar{x}\right) \quad \text { if } \bar{t} \geq \lambda_{2} \bar{x}, \quad \widetilde{g}_{2}\left(\bar{t}-\lambda_{2} \bar{x}\right)=0 \quad \text { if } \lambda_{2} \bar{x} \geq \bar{t} \geq \lambda_{1} \bar{x},
$$

and

$$
\widetilde{a}=0 \quad \text { if } \bar{t} \geq \lambda_{2} \bar{x}, \quad \widetilde{a}=\frac{\lambda_{2} \bar{x}-\bar{t}}{\lambda_{2}-\lambda_{1}} \quad \text { if } \lambda_{2} \bar{x} \geq \bar{t} \geq \lambda_{1} \bar{x} .
$$

Now, by substituting the expression $(47)_{3}$, i.e. the third equation of (47), for $\psi_{03}$ into the boundary condition $(46)_{3}$ we obtain the following integral equation

$$
a_{1}^{-1} \int_{\frac{\bar{t}}{2 \lambda_{1}}}^{0} q_{1}(\xi) d \xi+\lambda_{1} \int_{\frac{\bar{t}}{2 \lambda_{1}}}^{0} q_{1}(\xi)\left(\psi_{02}-\psi_{01}\right)\left(\xi,-\lambda_{1} \xi+\bar{t}\right) d \xi=\frac{d g_{1}(\bar{t})}{d \bar{t}} .
$$

To obtain a system of integral equations in standard form, it is convenient to define

$$
\varphi_{0 i}(\bar{x}, \bar{t}) \equiv \psi_{0 i, \bar{t}}(\bar{x}, \bar{t}), \quad i=1,4 .
$$

The integral equations for $\varphi_{0 i}$ can be formally derived by taking the derivative with respect to the time parameter $\bar{t}$ of the previous equations (47), (50), and recalling that $\varphi_{0 i}(\bar{x}, \bar{t})=0$ if $t=\lambda_{1} x$. In conclusion, we have the following system of five integral equations with unknowns $\left\{\varphi_{01}, \varphi_{02}, \varphi_{03}, \varphi_{04}, q_{2}\right\}$ (note that $q_{1}=-\rho_{1}^{-1} \rho_{2} q_{2}$ )

$$
\left\{\begin{array}{l}
\varphi_{01}(x, t)=g_{1, t}\left(t-\lambda_{1} x\right)+\int_{0}^{x} \varphi_{03}\left(\xi, \lambda_{1}(\xi-x)+t\right) d \xi, \\
\varphi_{02}(x, t)=\widetilde{g}_{2, t}\left(t-\lambda_{2} x\right)+\int_{\widetilde{a}}^{x} \varphi_{04}\left(\xi, \lambda_{2}(\xi-x)+t\right) d \xi, \\
\varphi_{03}(x, t)=\frac{1}{2} a_{1}^{-1} \rho_{1}^{-1} \rho_{2} q_{2}\left(\frac{\lambda_{1} x+t}{2 \lambda_{1}}\right)-\lambda_{1}^{2} \rho_{1}^{-1} \rho_{2} \int_{\frac{\lambda_{1} x+t}{2 \lambda_{1}}}^{x} q_{2}(\xi)\left(\varphi_{02}-\varphi_{01}\right)\left(\xi, \lambda_{1}(x-\xi)+t\right) d \xi, \\
\varphi_{04}(x, t)=-\frac{\lambda_{2}^{2} \lambda_{1}^{-1} a_{1}^{-1}}{\lambda_{1}+\lambda_{2}} q_{2}\left(\frac{\lambda_{2} x+t}{\lambda_{1}+\lambda_{2}}\right)+\lambda_{2}^{2} \int_{\frac{\lambda_{2} x+t}{\lambda_{1}+\lambda_{2}}}^{x} q_{2}(\xi)\left(\varphi_{02}-\varphi_{01}\right)\left(\xi, \lambda_{2}(x-\xi)+t\right) d \xi, \\
q_{2}\left(\frac{t}{2 \lambda_{1}}\right)=2 a_{1} \lambda_{1}^{2} \int_{\frac{t}{2 \lambda_{1}}}^{0} q_{2}(\xi)\left(\varphi_{02}-\varphi_{01}\right)\left(\xi,-\lambda_{1} \xi+t\right) d \xi+2 a_{1} \rho_{1} \rho_{2}^{-1} \lambda_{1} \frac{d^{2} g_{1}(t)}{d t^{2}},
\end{array}\right.
$$

where the variables $(\bar{x}, \bar{t})$ have been replaced by $(x, t)$ to simplify notation.

The system of integral equations (52) is closed in the triangular domain

$$
D^{T}=\left\{(x, t) \mid \lambda_{1} x \leq t \leq T-\lambda_{1} x, \quad 0 \leq x \leq X \equiv \frac{T}{2 \lambda_{1}}\right\} .
$$

In order to study the uniqueness and existence of the solution of (52) for "small" $X$, we define

$$
\left(v_{1}(x, t), v_{2}(x, t), v_{3}(x, t), v_{4}(x, t), v_{5}(x, t)\right)=\left(\varphi_{01}(x, t), \varphi_{02}(x, t), \varphi_{03}(x, t), \varphi_{04}(x, t), q_{2}(x)\right)
$$

and we rewrite the system in the following form

$$
\left\{\begin{array}{l}
v_{1}(x, t)=\zeta_{1}(x, t)+\int_{0}^{x} v_{3}\left(\xi, X_{1}(\xi ; x, t)\right) d \xi \\
v_{2}(x, t)=\zeta_{2}(x, t)+\int_{0}^{x} \chi_{1}^{*} v_{4}\left(\xi, X_{2}(\xi ; x, t)\right) d \xi \\
v_{3}(x, t)=k_{1} v_{5}\left(Y_{1}(x, t)\right)+\int_{0}^{Y_{1}(x, t)} \chi_{1}(\xi ; t, x) v_{5}(\xi)\left(v_{2}-v_{1}\right)\left(\xi, X_{3}(\xi ; t, x)\right) d \xi \\
v_{4}(x, t)=k_{2} v_{5}\left(Y_{2}(x, t)\right)+\int_{0}^{Y_{2}(x, t)} \chi_{2}(\xi ; t, x) v_{5}(\xi)\left(v_{2}-v_{1}\right)\left(\xi, X_{4}(\xi ; t, x)\right) d \xi \\
v_{5}(x)=\zeta_{3}(x)+k_{3} \int_{0}^{x} v_{5}(\xi)\left(v_{2}-v_{1}\right)\left(\xi ; X_{5}(\xi ; t)\right) d \xi
\end{array}\right.
$$


where $\zeta_{i} \in C^{0}\left(D^{T}\right), i=1,2 ; \zeta_{3} \in C^{0}([0, X]) ; X_{i}(\xi ; x, t) \in C^{0}\left([0, X] \times D^{T}\right), i=1,4 ; X_{5}(\xi ; t) \in$ $C^{0}([0, X] \times[0, T]) ; Y_{i}(x, t) \in C^{0}\left(D^{T}\right)$ with $0 \leq Y_{2}(x, t) \leq Y_{1}(x, t) \leq X, i=1,2 ; \chi_{1}^{*}(\cdot ; x, t)$, $\chi_{1}(\cdot ; x, t), \chi_{2}(\cdot ; x, t) \in L^{\infty}([0, X])$ are uniformly bounded and continuous with respect to the variables $(x, t)$ in $D^{T} ; k_{i}, i=1,3$, are known constants depending on $a_{1}, f_{1}, \rho_{1}, \rho_{2}, \lambda_{1}, \lambda_{2}$.

By expressing $v_{5}$ in $(55)_{3,4}$ using $(55)_{5}$, the previous system can be rewritten as

$$
\left\{\begin{array}{l}
v_{1}(x, t)=\zeta_{1}(x, t)+\int_{0}^{x} v_{3}\left(\xi, X_{1}(\xi ; x, t)\right) d \xi \\
v_{2}(x, t)=\zeta_{2}(x, t)+\int_{0}^{x} \chi_{1}^{*}(\xi ; x, t) v_{4}\left(\xi, X_{2}(\xi ; x, t)\right) d \xi \\
v_{3}(x, t)=k_{1} \zeta_{3}\left(Y_{1}(x, t)\right)+k_{1} k_{3} \int_{0}^{Y_{1}(x, t)} v_{5}(\xi)\left(v_{2}-v_{1}\right)\left(\xi ; X_{5}(\xi ; t)\right) d \xi+ \\
+\int_{0}^{Y_{1}(x, t)} \chi_{1}(\xi ; t, x) v_{5}(\xi)\left(v_{2}-v_{1}\right)\left(\xi, X_{3}(\xi ; t, x)\right) d \xi \\
v_{4}(x, t)=k_{2} \zeta_{3}\left(Y_{2}(x, t)\right)+k_{2} k_{3} \int_{0}^{Y_{2}(x, t)} v_{5}(\xi)\left(v_{2}-v_{1}\right)\left(\xi ; X_{5}(\xi ; t)\right) d \xi+ \\
+\int_{0}^{Y_{2}(x, t)} \chi_{2}(\xi ; t, x) v_{5}(\xi)\left(v_{2}-v_{1}\right)\left(\xi, X_{4}(\xi ; t, x)\right) d \xi \\
v_{5}(x)=\zeta_{3}(x)+k_{3} \int_{0}^{x} v_{5}(\xi)\left(v_{2}-v_{1}\right)\left(\xi ; X_{5}(\xi ; t)\right) d \xi .
\end{array}\right.
$$

Let us introduce the function space

$$
\Phi_{X}=\left\{v=\left(v_{1}, v_{2}, v_{3}, v_{4}, v_{5}\right) \mid v_{j} \in C^{0}\left(D^{T}\right), j=1,4, v_{5} \in C^{0}([0, X])\right\},
$$

endowed with the norm

$$
\|v\|=\sum_{j=1}^{4}\left\|v_{j}\right\|_{C^{0}\left(D^{T}\right)}+\left\|v_{5}\right\|_{C^{0}([0, X])} .
$$

Let $V_{X}: \Phi_{X} \rightarrow \Phi_{X}, V_{X}(v)=w$, be the operator defined as follows

$$
\left\{\begin{array}{l}
w_{1}(x, t)=f_{1}(x, t)+\int_{0}^{x} v_{3}\left(\xi, X_{1}(\xi ; x, t)\right) d \xi \\
w_{2}(x, t)=f_{2}(x, t)+\int_{0}^{x} \chi_{1}^{*}(\xi ; x, t) v_{4}\left(\xi, X_{2}(\xi ; x, t)\right) d \xi \\
w_{3}(x, t)=f_{3}(x, t)+k_{1} k_{3} \int_{0}^{Y_{1}(x, t)} v_{5}(\xi)\left(v_{2}-v_{1}\right)\left(\xi ; X_{5}(\xi ; t)\right) d \xi+ \\
+\int_{0}^{Y_{1}(x, t)} \chi_{1}(\xi ; t, x) v_{5}(\xi)\left(v_{2}-v_{1}\right)\left(\xi, X_{3}(\xi ; t, x)\right) d \xi \\
w_{4}(x, t)=f_{4}(x, t)+k_{2} k_{3} \int_{0}^{Y_{2}(x, t)} v_{5}(\xi)\left(v_{2}-v_{1}\right)\left(\xi ; X_{5}(\xi ; t)\right) d \xi+ \\
+\int_{0}^{Y_{2}(x, t)} \chi_{2}(\xi ; t, x) v_{5}(\xi)\left(v_{2}-v_{1}\right)\left(\xi, X_{4}(\xi ; t, x)\right) d \xi \\
w_{5}(x)=f_{5}(x)+k_{3} \int_{0}^{x} v_{5}(\xi)\left(v_{2}-v_{1}\right)\left(\xi ; X_{5}(\xi ; t)\right) d \xi
\end{array}\right.
$$

where $F:=\left(f_{1}, f_{2}, f_{3}, f_{4}, f_{5}\right)$ is given by

$$
F=\left(\zeta_{1}(x, t), \zeta_{2}(x, t), k_{1} \zeta_{3}\left(Y_{1}(x, t)\right), k_{2} \zeta_{3}\left(Y_{2}(x, t)\right), \zeta_{3}(x)\right) \neq(0,0,0,0,0), \quad F \in \Phi_{X}
$$

since $F$ contains derivatives of $g_{i}(t), i=1,2$ up to second order and $g_{i}(t), i=1,2$ are $C^{2}$.

It is easy to show that $V_{X} v \in \Phi_{X}$ for every $v \in \Phi_{X}$.

Let us define $B_{\gamma, X}(F)=\left\{v \in \Phi_{X} \mid\|v-F\| \leq \gamma\right\}$, with $\gamma>0$. To prove existence and uniqueness of the solution for the system (56) it is enough to show that: i) $\forall \gamma>0 \exists X>0$ (small enough) such that $V_{X}\left(B_{\gamma, X}(F)\right) \subset B_{\gamma, X}(F)$; ii) $\forall \gamma>0 \exists X>0$ (small enough) such that $\left\|V_{X}\left(v^{(1)}\right)-V_{X}\left(v^{(2)}\right)\right\| \leq \frac{1}{2}\left\|v^{(1)}-v^{(2)}\right\|$, for every $v^{(i)} \in \Phi_{X}, i=1,2$. 
To prove condition i), let $v \in B_{\gamma, X}(F)$. By (59) it is easy to show that

$$
\begin{gathered}
\sum_{j=1}^{4}\left|w_{j}-f_{j}\right|+\left|w_{5}-f_{5}\right| \leq \int_{0}^{x}\left|v_{3}\left(\xi, X_{1}(\xi ; x, t)\right)\right| d \xi+\int_{0}^{x}\left|v_{4}\left(\xi, X_{2}(\xi ; x, t)\right)\right| d \xi+ \\
\quad+\left|k_{1} k_{3}\right| \int_{0}^{Y_{1}(x, t)}\left|v_{5}(\xi)\right|\left|\left(v_{2}-v_{1}\right)\left(\xi ; X_{5}(\xi ; t)\right)\right| d \xi+ \\
+C_{1} \int_{0}^{Y_{1}(x, t)}\left|v_{5}(\xi)\right|\left|\left(v_{2}-v_{1}\right)\left(\xi, X_{3}(\xi ; t, x)\right)\right| d \xi+\left|k_{2} k_{3}\right| \int_{0}^{Y_{2}(x, t)}\left|v_{5}(\xi)\right|\left|\left(v_{2}-v_{1}\right)\left(\xi ; X_{5}(\xi ; t)\right)\right| d \xi+ \\
+C_{2} \int_{0}^{Y_{2}(x, t)}\left|v_{5}(\xi)\right|\left|\left(v_{2}-v_{1}\right)\left(\xi, X_{4}(\xi ; t, x)\right)\right| d \xi+\left|k_{3}\right| \int_{0}^{x}\left|v_{5}(\xi)\right|\left|\left(v_{2}-v_{1}\right)\left(\xi ; X_{5}(\xi ; t)\right)\right| d \xi \leq \\
\leq C_{3} X\left(\|v\|+\|v\|^{2}\right) \leq C_{4} X(\|F\|+\gamma)(1+\|F\|+\gamma), \quad(61)
\end{gathered}
$$

where $C_{i}, i=1,4$, are positive constants. Therefore, $V_{X} v \in B_{\gamma, X}(F)$ if $X$ is small enough, e.g. $X \leq \frac{\gamma}{C(\|F\|+\gamma)(1+\|F\|+\gamma)}$, where $C$ is a positive constant only depending on the data.

To prove condition ii), let us denote $z=V_{X} v^{(1)}-V_{X} v^{(2)}$. By (59) we have

$$
\left\{\begin{array}{l}
z_{1}(x, t)=\int_{0}^{x}\left(v_{3}^{(1)}-v_{3}^{(2)}\right)\left(\xi, X_{1}(\xi ; x, t)\right) d \xi \\
z_{2}(x, t)=\int_{0}^{x} \chi_{1}^{*}(\xi ; x, t)\left(v_{4}^{(1)}-v_{4}^{(2)}\right)\left(\xi, X_{2}(\xi ; x, t)\right) d \xi \\
z_{3}(x, t)=k_{1} k_{3} \int_{0}^{Y_{1}(x, t)}\left(v_{5}^{(1)}(\xi)\left(v_{2}^{(1)}-v_{1}^{(1)}\right)\left(\xi ; X_{5}(\xi ; t)\right)-v_{5}^{(2)}(\xi)\left(v_{2}^{(2)}-v_{1}^{(2)}\right)\left(\xi ; X_{5}(\xi ; t)\right)\right) d \xi+ \\
+\int_{0}^{Y_{1}(x, t)} \chi_{1}(\xi ; t, x)\left(v_{5}^{(1)}(\xi)\left(v_{2}^{(1)}-v_{1}^{(1)}\right)\left(\xi, X_{3}(\xi ; t, x)\right)-v_{5}^{(2)}(\xi)\left(v_{2}^{(2)}-v_{1}^{(2)}\right)\left(\xi, X_{3}(\xi ; t, x)\right)\right) d \xi, \\
z_{4}(x, t)=k_{2} k_{3} \int_{0}^{Y_{2}(x, t)}\left(v_{5}^{(1)}(\xi)\left(v_{2}^{(1)}-v_{1}^{(1)}\right)\left(\xi ; X_{5}(\xi ; t)\right)-v_{5}^{(2)}(\xi)\left(v_{2}^{(2)}-v_{1}^{(2)}\right)\left(\xi ; X_{5}(\xi ; t)\right)\right) d \xi+ \\
+\int_{0}^{Y_{2}(x, t)} \chi_{2}(\xi ; t, x)\left(v_{5}^{(1)}(\xi)\left(v_{2}^{(1)}-v_{1}^{(1)}\right)\left(\xi, X_{4}(\xi ; t, x)\right)-v_{5}^{(2)}(\xi)\left(v_{2}^{(2)}-v_{1}^{(2)}\right)\left(\xi, X_{4}(\xi ; t, x)\right)\right) d \xi, \\
z_{5}(x)=k_{3} \int_{0}^{x}\left(v_{5}^{(1)}(\xi)\left(v_{2}^{(1)}-v_{1}^{(1)}\right)\left(\xi ; X_{5}(\xi ; t)\right)-v_{5}^{(2)}(\xi)\left(v_{2}^{(2)}-v_{1}^{(2)}\right)\left(\xi ; X_{5}(\xi ; t)\right)\right) d \xi .
\end{array}\right.
$$

From $(62)_{1,2}$ we have

$$
\left\|z_{i}\right\| \leq X\left\|v^{(1)}-v^{(2)}\right\|, \quad i=1,2
$$

By observing that

$$
\begin{aligned}
v_{5}^{(1)}\left(v_{2}^{(1)}-v_{1}^{(1)}\right)- & v_{5}^{(2)}\left(v_{2}^{(2)}-v_{1}^{(2)}\right)= \\
& v_{5}^{(1)}\left(v_{2}^{(1)}-v_{2}^{(2)}\right)+v_{2}^{(2)}\left(v_{5}^{(1)}-v_{5}^{(2)}\right)+v_{5}^{(2)}\left(v_{1}^{(2)}-v_{1}^{(1)}\right)+v_{1}^{(1)}\left(v_{5}^{(2)}-v_{5}^{(1)}\right),
\end{aligned}
$$

the products of terms appearing in equations $(62)_{3-5}$ can be bounded as follows

$$
\left\|z_{i}\right\| \leq C X(\|F\|+\gamma)\left\|v^{(1)}-v^{(2)}\right\|, \quad i=3,4,5,
$$

where $C$ is a positive constant only depending on the data. Therefore, condition ii) is satisfied provided that we choose $X$ small enough, e.g. $X \leq \frac{1}{2(1+C(\|F\|+\gamma))}$. Choosing $\gamma=\|F\|$, it is enough to take

$$
X=\min \left(\frac{1}{2 C(1+2\|F\|)}, \frac{1}{2(1+2 C\|F\|)}\right)
$$


to satisfy the conditions i) and ii). Hence we have proven the proposition.

\section{Justification of the Layer Stripping method.}

Let us now justify that we can reach any point $c$ in $(0, L)$ in finitely many steps.

Indeed, let $c_{i} \in(0, L)$ be the sequence of points given by the Layer Stripping. We know that

$$
c_{i}=\min \left(\frac{1}{2 C\left(1+2\left\|F_{i}\right\|\right)}, \frac{1}{2\left(1+2 C\left\|F_{i}\right\|\right)}\right)
$$

where $C$ depends on the bounds of the coefficients on $(0, L)$.

Hence, it is enough to prove that the sequence $\left\|F_{i}\right\|$ is bounded. To justify this, we proceed as follows:

We know that $f_{i}$ is defined on $\left(c_{i}, L\right)$ by the first and the second derivatives of $R^{T}(\delta(t), 0)$ evaluated at $x:=c_{i}$ which is given by $g_{i}(t)=u^{\delta}\left(c_{i}, t\right)$. In every step of the Layer Stripping we take $T<\lambda_{1}\left(L-c_{i}\right)$ to avoid the reflections.

We use the change of variable $y:=\frac{x}{c_{i}}$ to reduce the problem on $\left(1, \frac{L}{c_{i}}\right)$ and the change of variables $z:=y-1$ to reduce the problem on $\left(0, \frac{L}{c_{i}}-1\right)$. We can replace $\frac{L}{c_{i}}-1$ by $L^{\prime}$ large enough and independent on $c_{i}$. This is justified by the finite velocity of propagation.

Of course the coefficients of the new problems are depending on $i$. But all these changes of variables are linear hence they preserve the boundness of these coefficients with respect to $i$.

Now we are dealing with the same hyperbolic problem as the (16) with a sequence of coefficients bounded with respect to $i$, zero initial conditions and zero Dirichlet on $z=L^{\prime}$ and on the boundary $x=0$ we have as a Neumann data the delta function.

Let us prove that the corresponding sequence $\left\|F_{i}\right\|$ is bounded or, by definition of $f_{i}$, that the sequences given by the zero, the first and second derivatives of $g_{i}$ are bounded with respect to the maximum norm. We recall that $g_{i}$ is the trace on $z=0$ of the solution of the above hyperbolic problem.

Looking for the solution as a sum of singular and regular parts, we deduce that the singular part has the required property since it given by (28). We have to prove the same for the regular one. Recall that this regular part satisfies the hyperbolic problem with homogenous initial and boundary conditions and an $L^{2}\left((0, T) \times\left(0, L^{\prime}\right)\right)$ second member as in (30). From the general theory [Lad] or [W], we know that this sequence, indexed by $i$, is bounded in the energy norm i.e $C\left([0, T], H^{1}\left(0, L^{\prime}\right)\right)$ and in $C^{1}\left([0, T], L^{2}\left(0, L^{\prime}\right)\right)$ since the sequence of coefficients is uniformly bounded. Hence we deduce that its trace on $z=0$, i.e $g_{i}^{r}:=\left(g_{i, 1}^{r}, g_{i, 2}^{r}\right)$ (r: for regular) is bounded in $C[0, T]$.

We repeat the computations done in the proof of Proposition 3.5 replacing the previous coefficients by the new sequences of coefficients. From the equation (50), we deduce that the first derivative of $g_{i, 1}^{r}$ is bounded.

Since $u_{i}^{r}$ is bounded in $C^{1}\left([0, T], L^{2}\left(0, L^{\prime}\right)\right)$, we have $\frac{d}{d_{t}} u_{i, 1}^{r}:=\varphi_{0,1}$ and $\frac{d}{d_{t}} u_{i, 2}^{r}:=\varphi_{0,2}$ are bounded in $L^{2}\left((0, T) \times\left(0, L^{\prime}\right)\right)$. Hence from the fifth equation of $(52)$, we deduce that the second derivative of $g_{i, 1}^{r}$ is bounded.

Using similar equations for $g_{i, 2}^{r}$ as it was done for $g_{i, 1}^{r}$, we get the corresponding equation (50) and the fifth equation of (52) for $g_{i, 2}^{r}$. 
Arguing as for $g_{i, 1}^{r}$, see above, we deduce that the first and the second derivatives of $g_{i, 2}^{r}$ are also bounded. This ends the justification.

Proof of Proposition 3.6. We start by taking $\omega \in \mathbb{C}$ such that $\omega^{2} \in \mathbb{C} \backslash \mathbb{R}^{+}$. After knowing $\Lambda_{\omega}^{(c, L)}$ for these frequencies, we shall proceed by approximation to reconstruct $\Lambda_{\omega}^{(c, L)}$ for $\omega^{2} \in$ $\mathbb{R}^{+} \backslash \sigma^{(0, L)}$.

Let $V=\left(V_{1}(x), V_{2}(x)\right)$ solves the problem

$$
\left\{\begin{array}{l}
-A V_{, x x}+K(x) V-\omega^{2} R V=0 \quad x \in(c, L), \\
\left.A V_{, x}\right|_{x=c}=a \\
\left.V\right|_{x=L}=0
\end{array}\right.
$$

where $a=a_{1} e_{1}+a_{2} e_{2}$ with $a_{1}, a_{2} \in \mathbb{R}, e_{1}=(1,0)$ and $e_{2}=(0,1)$.

We wish to determine $\left.V\right|_{x=c}=\Lambda_{\omega}^{(c, L)}\left(\begin{array}{l}a \\ 0\end{array}\right)$ from $\Lambda_{\omega}^{(0, L)}$ and the knowledge of the coefficient $k$ in $(0, c), c<L$.

Let $\phi$ be the solution of the Cauchy problem

$$
\left\{\begin{array}{l}
-A \phi_{, x x}+K(x) \phi-\omega^{2} R \phi=0 \quad x \in(0, c), \\
\left.A \phi_{, x}\right|_{x=c}=a \\
\left.\phi\right|_{x=c}=\left.V\right|_{x=c}
\end{array}\right.
$$

We define $\left.V\right|_{x=c}=\left(v_{1}, v_{2}\right)$. Therefore, the function $\phi$ can be written as

$$
\phi=\phi_{0}+v_{1} \phi_{1}+v_{2} \phi_{2},
$$

where $\phi_{0}=\phi\left(\begin{array}{c}a \\ 0\end{array}\right), \phi_{1}=\phi\left(\begin{array}{c}0 \\ e_{1}\end{array}\right), \phi_{2}=\phi\left(\begin{array}{c}0 \\ e_{2}\end{array}\right)$ and where $\phi\left(\begin{array}{c}X \\ Y\end{array}\right)$ is the solution of (68) replacing $a$ and $\left.V\right|_{x=c}$ by $X$ and $Y$, respectively.

Let us introduce

$$
U=\left\{\begin{array}{l}
\phi \text { in }(0, c] \\
V \text { in }[c, L)
\end{array}\right.
$$

The function $U$ satisfies the problem

$$
\left\{\begin{array}{l}
-A U_{, x x}+K(x) U-\omega^{2} R U=0 \quad x \in(0, L), \\
\left.A U_{, x}\right|_{x=0}=\left.\phi_{, x}\right|_{x=0}, \\
\left.U\right|_{x=L}=0
\end{array}\right.
$$

since $\left.V\right|_{x=c}=\left.\phi\right|_{x=c}$ and $\left.V_{x}\right|_{x=c}=\left.\phi_{, x}\right|_{x=c}$. Moreover, since $\left.U\right|_{x=c}=\left.V\right|_{x=c}$, the restriction of $U$ on the interval $(0, c)$ satisfies

$$
\left\{\begin{array}{l}
-A U_{, x x}+K(x) U-\omega^{2} R U=0 \quad x \in(0, c), \\
\left.A U_{, x}\right|_{x=0}=\bar{\alpha}_{0}+v_{1} \bar{\alpha}_{1}+v_{2} \bar{\alpha}_{2}, \\
\left.U\right|_{x=c}=v_{1} e_{1}+v_{2} e_{2},
\end{array}\right.
$$

where $\bar{\alpha}_{i}=\left.\phi_{i, x}\right|_{x=0}, i=0,1,2$. 
From (69) and (70) we deduce that

$$
\Lambda_{\omega}^{(0, c)}\left(\begin{array}{c}
\bar{\alpha}_{0}+v_{1} \bar{\alpha}_{1}+v_{2} \bar{\alpha}_{2} \\
v_{1} e_{1}+v_{2} e_{2}
\end{array}\right)=\Lambda_{\omega}^{(0, L)}\left(\begin{array}{c}
\bar{\alpha}_{0}+v_{1} \bar{\alpha}_{1}+v_{2} \bar{\alpha}_{2} \\
0
\end{array}\right)
$$

By linearity of the Neumann-to-Dirichlet operators we have

$$
\Lambda_{\omega}^{(0, c)}\left(\begin{array}{c}
\bar{\alpha}_{0}+v_{1} \bar{\alpha}_{1}+v_{2} \bar{\alpha}_{2} \\
v_{1} e_{1}+v_{2} e_{2}
\end{array}\right)=\Lambda_{\omega}^{(0, c)}\left(\begin{array}{c}
\bar{\alpha}_{0} \\
0
\end{array}\right)+v_{1} \Lambda_{\omega}^{(0, c)}\left(\begin{array}{c}
\bar{\alpha}_{1} \\
e_{1}
\end{array}\right)+v_{2} \Lambda_{\omega}^{(0, c)}\left(\begin{array}{c}
\bar{\alpha}_{2} \\
e_{2}
\end{array}\right)
$$

and

$$
\Lambda_{\omega}^{(0, L)}\left(\begin{array}{c}
\bar{\alpha}_{0}+v_{1} \bar{\alpha}_{1}+v_{2} \bar{\alpha}_{2} \\
0
\end{array}\right)=\Lambda_{\omega}^{(0, L)}\left(\begin{array}{c}
\bar{\alpha}_{0} \\
0
\end{array}\right)+v_{1} \Lambda_{\omega}^{(0, L)}\left(\begin{array}{c}
\bar{\alpha}_{1} \\
0
\end{array}\right)+v_{2} \Lambda_{\omega}^{(0, L)}\left(\begin{array}{c}
\bar{\alpha}_{2} \\
0
\end{array}\right) .
$$

Therefore, from (71) we have

$$
\begin{gathered}
v_{1}\left(\Lambda_{\omega}^{(0, c)}\left(\begin{array}{c}
\bar{\alpha}_{1} \\
e_{1}
\end{array}\right)-\Lambda_{\omega}^{(0, L)}\left(\begin{array}{c}
\bar{\alpha}_{1} \\
0
\end{array}\right)\right)+v_{2}\left(\Lambda_{\omega}^{(0, c)}\left(\begin{array}{c}
\bar{\alpha}_{2} \\
e_{2}
\end{array}\right)-\Lambda_{\omega}^{(0, L)}\left(\begin{array}{c}
\bar{\alpha}_{2} \\
0
\end{array}\right)\right)= \\
=\left(\Lambda_{\omega}^{(0, L)}-\Lambda_{\omega}^{(0, c)}\right)\left(\begin{array}{c}
\bar{\alpha}_{0} \\
0
\end{array}\right)
\end{gathered}
$$

This last system can be written as

$$
\left(\begin{array}{ll}
B_{1} & C_{1} \\
B_{2} & C_{2}
\end{array}\right)\left(\begin{array}{l}
v_{1} \\
v_{2}
\end{array}\right)=\left(\begin{array}{l}
f_{1} \\
f_{2}
\end{array}\right)
$$

where

$$
B=\left(\begin{array}{c}
B_{1} \\
B_{2}
\end{array}\right)=\Lambda_{\omega}^{(0, c)}\left(\begin{array}{c}
\bar{\alpha}_{1} \\
e_{1}
\end{array}\right)-\Lambda_{\omega}^{(0, L)}\left(\begin{array}{c}
\bar{\alpha}_{1} \\
0
\end{array}\right)
$$

and

$$
C=\left(\begin{array}{c}
C_{1} \\
C_{2}
\end{array}\right)=\Lambda_{\omega}^{(0, c)}\left(\begin{array}{c}
\bar{\alpha}_{2} \\
e_{2}
\end{array}\right)-\Lambda_{\omega}^{(0, L)}\left(\begin{array}{c}
\bar{\alpha}_{2} \\
0
\end{array}\right) .
$$

Let us now prove that the system (75) is solvable. It is enough to prove that the vectors $B$ and $C$ are linearly independent.

Let us consider $(x, y) \in \mathbb{R}^{2}$ such that $x B+y C=0$. We have

$$
\Lambda_{\omega}^{(0, c)}\left(\begin{array}{c}
x \bar{\alpha}_{1} \\
x e_{1}
\end{array}\right)-\Lambda_{\omega}^{(0, L)}\left(\begin{array}{c}
x \bar{\alpha}_{1} \\
0
\end{array}\right)+\Lambda_{\omega}^{(0, c)}\left(\begin{array}{c}
y \bar{\alpha}_{2} \\
y e_{2}
\end{array}\right)-\Lambda_{\omega}^{(0, L)}\left(\begin{array}{c}
y \bar{\alpha}_{2} \\
0
\end{array}\right)=0
$$

or, equivalently,

$$
\Lambda_{\omega}^{(0, c)}\left(\begin{array}{c}
x \bar{\alpha}_{1}+y \bar{\alpha}_{2} \\
x e_{1}+y e_{2}
\end{array}\right)-\Lambda_{\omega}^{(0, L)}\left(\begin{array}{c}
x \bar{\alpha}_{1}+y \bar{\alpha}_{2} \\
0
\end{array}\right)=0 .
$$

We write

$$
\left.f\right|_{x=0}=\Lambda_{\omega}^{(0, c)}\left(\begin{array}{c}
x \bar{\alpha}_{1}+y \bar{\alpha}_{2} \\
x e_{1}+y e_{2}
\end{array}\right) \text { and }\left.g\right|_{x=0}=\Lambda_{\omega}^{(0, L)}\left(\begin{array}{c}
x \bar{\alpha}_{1}+y \bar{\alpha}_{2} \\
0
\end{array}\right)
$$


with $f$ and $g$ satisfying

$$
\left\{\begin{array}{l}
-A f_{, x x}+K(x) f-\omega^{2} R f=0 \quad x \in(0, c), \\
\left.A f_{, x}\right|_{x=0}=x \bar{\alpha}_{1}+y \bar{\alpha}_{2} \\
\left.f\right|_{x=c}=x e_{1}+y e_{2}
\end{array}\right.
$$

and

$$
\left\{\begin{array}{l}
-A g_{, x x}+K(x) g-\omega^{2} R g=0 \quad x \in(0, L), \\
\left.A g_{, x}\right|_{x=0}=x \bar{\alpha}_{1}+y \bar{\alpha}_{2}, \\
\left.g\right|_{x=L}=0 .
\end{array}\right.
$$

From (76), we deduce that $f=g$ in $(0, c)$ since these functions satisfy the same equation in $(0, c)$ and have the same Cauchy data at $x=0$. Recalling that $\bar{\alpha}_{i}=\left.\phi_{i, x}\right|_{x=0}$, we have $x \bar{\alpha}_{1}+y \bar{\alpha}_{2}=\left.x \phi_{1, x}\right|_{x=0}+\left.y \phi_{2, x}\right|_{x=0}$.

Let us define $\Phi=x \phi_{1}+y \phi_{2}$. Then $\Phi=\phi\left(\begin{array}{c}0 \\ x e_{1}+y e_{2}\end{array}\right)$ satisfies

$$
\left\{\begin{array}{l}
-A \Phi_{, x x}+K(x) \Phi-\omega^{2} R \Phi=0 \quad x \in(0, c) \\
\left.A \Phi_{, x}\right|_{x=0}=x \bar{\alpha}_{1}+y \bar{\alpha}_{2} \\
\left.\Phi\right|_{x=c}=x e_{1}+y e_{2}
\end{array}\right.
$$

This means that $\Psi=f-\Phi$ satisfies

$$
\left\{\begin{array}{l}
-A \Psi_{, x x}+K(x) \Psi-\omega^{2} R \Psi=0 \quad x \in(0, c), \\
\left.A \Psi_{, x}\right|_{x=0}=0 \\
\left.\Psi\right|_{x=L}=0
\end{array}\right.
$$

then $\Psi=0$ and $f=\Phi$ in $(0, c)$, hence $g=\Phi$ in $(0, c)$.

By definition, $\Phi$ satisfies $\left.A \Phi_{, x}\right|_{x=c}=0$, then $\left.A g_{, x}\right|_{x=c}=0$ and hence $g$ satisfies

$$
\left\{\begin{array}{l}
-A g_{, x x}+K(x) g-\omega^{2} R g=0 \quad x \in(c, L), \\
\left.A g_{, x}\right|_{x=c}=0 \\
\left.g\right|_{x=L}=0
\end{array}\right.
$$

This implies that $g \equiv 0$ in $(c, L)$ since $\omega^{2}$ is not in $\mathbb{R}$, knowing that the spectrum of the problem $(77)$ is in $\mathbb{R}$. By continuation $g \equiv 0$ in $(0, L)$ and therefore $\Phi=0$ in $(0, c)$. This implies $(x, y)=(0,0)$, that is the linear independence of the vectors $B$ and $C$.

Finally, to determine $\left.V\right|_{x=c}=\left(v_{1}, v_{2}\right)$ it is enough to solve the linear system (75). Remark that the components of the vectors $B$ and $C$ can be computed from $\Lambda_{\omega}^{(0, L)}, \Lambda_{\omega}^{(0, c)}$ and from the solution of the Cauchy problem (68), which are all known.

\section{Concluding remarks}

In this paper we have considered the inverse problem of identifying the shearing stiffness coefficient of a steel-concrete composite beam from dynamical data. We have presented a global 
reconstruction procedure for determining the unknown coefficient based on measurements of the dynamical Neumann-to-Dirichlet operator related to the longitudinal motions of the beam.

We wish to conclude with some remarks about open problems and possible directions of future research.

1) We have already mentioned in the Introduction that the small free vibrations of a composite beam are governed by a differential system where a coupling takes place between longitudinal and bending motions, see equation (1). In this paper we have focused our attention on the simplified model where the coupling is neglected and only longitudinal motions are present, see equation (4). Engineering applications suggest that it might be worth examining the full coupled case, which includes a fourth order operator and two second order operators. In this case it seems reasonable to consider the full $3 \times 3$ frequency response function at one end of the beam as dynamic data for the reconstruction of the shearing stiffness of the connection.

2) The identification procedure that has been discussed here is constructive, that is, starting from the measured data, the unknown coefficient is, in principle, computable. It would be interesting to verify, through numerical simulations, the efficiency of this reconstruction procedure and its sensitivity to measurement errors, in order to check the usefulness of the proposed diagnostic technique in practical real life applications.

ACKNOWLEDGMENT The first author wishes to thank Gen Nakamura and Mourad Sini for supporting his visit at the Hokkaido University and for the warm hospitality at the Department of Mathematics.

\section{References}

[B] M.I. Belishev, On relations between spectral and dynamical inverse data, Journal of Inverse and Ill-Posed Problems 6(9) (2001), 547-565.

[BA] M.I. Belishev and S.A. Avdonin, Local uniqueness in the dynamical inverse problem for a two velocity system, Journal of Mathematical Sciences 117 (2) (2003) 3910-3917.

[BMW] G. Biscontin, A. Morassi and P. Wendel, Vibrations of steel-concrete composite beams, Journal of Vibration and Control 6(5) (2000) 691-714.

[Bla] A.S. Blagoveshchenskii, On the inverse problem of the theory of seismic wave propagation. In Problems of Mathematical Physics (in Russian), LGU, Leningrad, issue 1, 68-91, 1966.

[DM] M. Dilena and A. Morassi, A damage analysis of steel-concrete composite beams via dynamic methods: Part II. Analytical models and damage detection, Journal of Vibration and Control 9(5) (2003) 529-565.

[E] D.J. Ewins, Modal testing: theory and practice, Research Studies Press, New York, 1984.

[J] R.P. Johnson, Composite structures of steel and concrete, Blackwell Scientific, Oxford, 1994. 
[KKL] A. Katchalov, Y. Kurylev and M. Lassas, Inverse boundary spectral problems, Chapman/CRC, Boca Raton, 2001.

[Lad] O.A. Ladyzhenskaya, The Boundary Value Problems of Mathematical Physics, Applied Mathematical Sciences Series 49, Springer-Verlag, New-York-Berlin, 1985.

[MR] A. Morassi and L. Rocchetto, A damage analysis of steel-concrete composite beams via dynamic methods: Part II. Experimental results, Journal of Vibration and Control 9(5) (2003) 507-527.

[Rom] V.G. Romanov, Inverse Problems of Mathematical Physics, VNU Sciences Press, Utrecht, 1987.

[Si] M. Sini, Some uniqueness results of discontinuous coefficients for the one dimensional inverse spectral problem, Inverse Problems 19 (2003) 871-894.

[W] J. Wloka, Partial differential equations, Cambridge University Press, 1987. 